\title{
Icosahedral invariants and a construction of class fields via periods of $K 3$ surfaces
}

\author{
Atsuhira Nagano
}

September 16, 2018

\begin{abstract}
In the theory of complex multiplication, it is important to construct class fields over CM fields. In this paper, we consider explicit $K 3$ surfaces parametrized by Klein's icosahedral invariants. Via the periods and the Shioda-Inose structures of $K 3$ surfaces, the special values of icosahedral invariants generate class fields over quartic CM fields. Moreover, we give an explicit expression of the canonical model of the Shimura variety for the simplest case via the periods of $K 3$ surfaces.
\end{abstract}

॥॥

\section{Introduction}

The aim of this paper is to construct class fields via $K 3$ surfaces. We obtain explicit class fields using the period mapping for a family of $K 3$ surfaces parametrized by Klein's icosahedral invariants of $\underline{\mathrm{K}}$.

In number theory, it is very interesting to find good special functions whose special values generate class fields (cf. Hilbert's 12th problem). Among such functions, the elliptic $j$-function is the most famous one. For an elliptic curve $E\left(g_{2}: g_{3}\right): y^{2}=4 x^{3}-g_{2} x-g_{3}$, the $j$-function is defined by the weighted quotient $j(z)=\frac{g_{2}^{3}}{g_{2}^{3}-27 g_{3}^{2}}$, where $z \in \mathbb{H}$ is the period of $E\left(g_{2}: g_{3}\right)$. If $E\left(g_{2}: g_{3}\right)$ has complex multiplication by an imaginary quadratic field $K$, it is well-known that $K=\mathbb{Q}(z)$ and the absolute class field of $K$ is given by $K(j(z))$ (Kronecker's Jugendtraum).

In the 20th century, A. Weil, G. Shimura, Y. Taniyama and many other researchers studied class fields using the modular functions coming from the moduli of abelian varieties with complex multiplication. Their theory is celebrated. However, since higher dimensional abelian varieties are defined by complicated equations, such modular functions are given by complicated forms (see [Mum] and [Gj]). So, it is much more difficult than the classical story of the elliptic $j$-function to construct explicit class fields via abelian varieties.

In this paper, we give a construction of class fields using not only abelian varieties but also $K 3$ surfaces. A $K 3$ surface is a 2-dimensional complex surface with the unique holomorphic 2-form up to a constant factor. So, a $K 3$ surface can be regarded as a 2-dimensional counterpart of an elliptic curve. Moreover, algebraic $K 3$ surfaces are sometimes defined by very simple equations. In this paper, we consider the $K 3$ surface $S(\mathfrak{A}: \mathfrak{B}: \mathfrak{C}): z^{2}=x^{3}-4\left(4 y^{3}-5 \mathfrak{A} y^{2}\right) x^{2}+20 \mathfrak{B} y^{3} x+\mathfrak{C} y^{4}$, where $\mathfrak{A}, \mathfrak{B}$ and $\mathfrak{C}$ are Klein's icosahedral invariants. The period of $S(\mathfrak{A}: \mathfrak{B}: \mathfrak{C})$ is given by a point $\left(z_{1}, z_{2}\right) \in \mathbb{H} \times \mathbb{H}$. The weighted quotients $X=\frac{\mathfrak{B}}{\mathfrak{A l}^{3}}, Y=\frac{\mathfrak{C}}{\mathfrak{l}^{5}}$ can be regarded as the Hilbert modular functions on $\mathbb{H} \times \mathbb{H}$ for $\mathbb{Q}(\sqrt{5})$, which is the real quadratic field with the smallest discriminant. We will see that $X, Y$ functions generate class fields over quartic CM fields (Theorem 2.1). So, we can regard the pair of our $X, Y$ functions as a very natural and simple counterpart of the elliptic $j$-function (see Table 1). Here, we note that the proof of this result is based on techniques for elliptic $K 3$ surfaces.

Keywords: class fields ; K3 surfaces ; Shimura varieties ; abelian varieties ; complex multiplication ; Hilbert modular functions ; quartic fields.

Mathematics Subject Classification 2010: Primary 11G45 ; Secondary 14J28, 14G35, 11F46, 11G15, $11 \mathrm{R} 16$.

Running head: Icosahedral invariants and class fields 


\begin{tabular}{ccc}
\hline & Classical Theory & Our Story \\
\hline Variety & Elliptic curve $E\left(g_{2}: g_{3}\right)$ & $K 3$ surface $S(\mathfrak{A}: \mathfrak{B}: \mathfrak{C})$ \\
Modular Function & $j=\frac{g_{2}^{3}}{g_{2}^{3}-27 g_{3}^{2}}$ on $\mathbb{H}$ & $X=\frac{\mathfrak{B}}{\mathfrak{A}^{3}}, Y=\frac{\mathfrak{C}}{\mathfrak{A l}^{5}}$ on $\mathbb{H} \times \mathbb{H}$ \\
Totally Real Field & $\mathbb{Q}$ & $\mathbb{Q}(\sqrt{5})$ \\
Class Field & $K(j) / K$ & $K^{*}(X, Y) / K^{*}$ \\
\hline
\end{tabular}

Table 1: The elliptic $j$-function and our $X, Y$ functions

This paper is organized as follows.

In Section 1, we recall class fields from abelian varieties with complex multiplication. We use these results in our argument.

In Section 2, we will see that $X, Y$ functions generate the unramified class fields $K^{*}(X, Y) / K^{*}$ for certain quartic CM fields $K^{*}$ (Theorem 2.1). The $X, Y$ functions are given by the inverse correspondence of the period mapping of our $K 3$ surface $S(\mathfrak{A}: \mathfrak{B}: \mathfrak{C})$. The Shioda-Inose structure connects the period mapping of $K 3$ surfaces and the moduli of abelian surfaces. Moreover, we obtain an explicit expression of the canonical model of the Hilbert modular surface as a Shimura variety for the real quadratic field of the smallest discriminant (Theorem 2.3). We note that such canonical models were firstly studied in [S3]. But, it is non-trivial to obtain an explicit expression for them. In Section 2, we obtain an explicit model derived from the period mapping of our $K 3$ surfaces. This result is given as a compilation of the previous works [N1, N. and [N3].

By the way, it is difficult to determine the Galois group for a class field, when the Galois group is not equal to the ideal class group. In fact, the Galois groups for our class fields $K^{*}(X, Y) / K^{*}$ do not always coincide with the ideal class groups of $K^{*}$. In Section 3, we determine the structure of the Galois groups $\operatorname{Gal}\left(K^{*}(X, Y) / K^{*}\right)$ for cyclic extensions $K / \mathbb{Q}$ (Theorem 3.1). Our result relies upon the result of Shimura S4 for cyclic extensions. The results in this section may be applied to the theory of cryptography, because explicit and detailed constructions of class fields are useful in that theory.

Our story gives a visible model of the sophisticated theory of class fields and Shimura varieties. The author expects that some explicit algebraic $K 3$ surfaces (for example, those studied in [CD], [EK], etc.) have direct applications in algebraic number theory via periods. The argument in this paper gives a prototype of such mathematical approaches. Moreover, $K 3$ surfaces have some merits, for they are studied from various viewpoints. For example, $K 3$ surfaces are well-studied in mirror symmetry. In fact, our $X, Y$ functions are closely related to mirror symmetry (see Remark 2.5). The author believes that our story concretely gives a new non-trivial relation between number theory and mirror symmetry.

\section{Abelian varieties with complex multiplication and class fields}

In this section, we survey the theory of abelian varieties with complex multiplication. For detail, see Shimura [S5]. The results in this section shall be used in Section 2 and 3.

\subsection{CM fields and CM types}

Let $K_{0}$ be a totally real number field such that $\left[K_{0}: \mathbb{Q}\right]=n$. A totally imaginary quadratic extension $K$ of $K_{0}$ is called a CM field. Let $\mathfrak{O}_{K}$ be the ring of integers of $K$. We have $2 n$ embeddings $\varphi_{1}, \cdots, \varphi_{2 n}: K \hookrightarrow$ C. We can suppose that $\left.\varphi_{1}\right|_{K_{0}}, \cdots,\left.\varphi_{n}\right|_{K_{0}}$ give distinct $n$ embeddings $K_{0} \hookrightarrow \mathbb{R}$ and $\varphi_{n+j}(j=1, \cdots, n)$ is complex conjugate of $\varphi_{j}$. We call $\left(K,\left\{\varphi_{1}, \cdots, \varphi_{n}\right\}\right)=\left(K,\left\{\varphi_{j}\right\}\right)$ a CM type.

For an abelian variety $A$ defined over $\mathbb{C}$, let $\operatorname{End}(A)$ be the ring of endomorphisms of $A$ and $\operatorname{End}_{0}(A)=$ $\operatorname{End}(A) \otimes_{\mathbb{Z}} \mathbb{Q}$. For an $n$-dimensional abelian variety $A$ and an embedding $\iota: K \hookrightarrow \operatorname{End}_{0}(A)$ such that

$\iota \circ i d_{K}=i d_{\operatorname{End}_{0}(\mathrm{~A})}$, we call $(A, \iota)$ an abelian variety of type $K$. Moreover, for the analytic representation $S$ of $\operatorname{End}_{0}(A)$ and the complex conjugate $\rho$, if $S\left(S^{\rho}\right.$, resp. $)$ is equivalent to the direct sum of $\varphi_{1}, \cdots, \varphi_{n}$ 
$\left(\varphi_{n+1}, \cdots, \varphi_{2 n}\right.$, resp. $),(A, \iota)$ is called an abelian variety of type $\left(K,\left\{\varphi_{j}\right\}\right)$. If $\left(A_{1}, \iota_{1}\right)$ and $\left(A_{2}, \iota_{2}\right)$ are abelian varieties of the same type, $A_{1}$ and $A_{2}$ are isogenous to each other.

From a given CM type, we can obtain a corresponding abelian variety as follows. For $\alpha \in K$, we set $u(\alpha)={ }^{t}\left(\alpha^{\varphi_{1}}, \cdots, \alpha^{\varphi_{n}}\right) \in \mathbb{C}^{n}$. Let $M \subset K$ be a free $\mathbb{Z}$-module of rank $2 n$. Let $\alpha_{1}, \cdots, \alpha_{2 n}$ be a system of basis of $M$. Then, the vectors $u\left(\alpha_{1}\right), \cdots, u\left(\alpha_{2 n}\right)$ span a lattice $\Lambda=\Lambda(M)$ of $\mathbb{C}^{n}$. Take $\zeta \in K$ such that $K=K_{0}(\zeta), \zeta^{2} \in K_{0},-\zeta^{2}$ is totally positive and $\operatorname{Im}\left(\zeta^{\varphi_{j}}\right)>0$ for $j \in\{1, \cdots, n\}$. Set

$$
E(z, w)=\sum_{j=1}^{n} \zeta^{\varphi_{j}}\left(z_{j} \overline{w_{j}}-\overline{z_{j}} w_{j}\right)
$$

for $z=\left(z_{1}, \cdots, z_{n}\right), w=\left(w_{1}, \cdots, w_{n}\right) \in \mathbb{C}^{n}$. We can check that $E(z, \sqrt{-1} w)=-\sqrt{-1} \sum_{j=1}^{n} \zeta^{\varphi_{j}}\left(z_{j} \overline{w_{j}}+\right.$ $\left.\overline{z_{j}} w_{j}\right)$ is symmetric and positive non-degenerate. We obtain $E(u(\alpha), u(\beta))=\operatorname{Tr}_{K / \mathbb{Q}}\left(\zeta \alpha \beta^{\rho}\right)$ for $\alpha, \beta \in K$. We can find a positive integer $\nu$ such that $\nu E(z, w)$ defines a non-degenerate Riemann form on $\mathbb{C}^{n} / \Lambda(M)$. Therefore, the complex torus $\mathbb{C}^{n} / \Lambda$ gives an n-dimensional abelian variety $A=A(M)$. For $\alpha \in K$, set $q(\alpha)=\left(\begin{array}{ccc}\alpha^{\varphi_{1}} & & 0 \\ & \cdots & \\ 0 & & \alpha^{\varphi_{n}}\end{array}\right)$. The linear transformation given by $q(\alpha)$ defines $\iota(\alpha) \in \operatorname{End}_{0}(A(M))$. So, $(A(M), \iota)$ is an abelian variety of type $\left(K,\left\{\varphi_{j}\right\}\right)$.

The above $(A(M), \iota)$ satisfies $\iota(K) \subset \operatorname{End}_{0}(A(M))$. However, we cannot assure that

$$
\iota\left(\mathfrak{O}_{K}\right)=\operatorname{End}(A(M)) \cap \iota(K) .
$$

Definition 1.1. We call the pair $(A, \iota)$ satisfying (1.2) an abelian variety of CM type $\left(\mathfrak{O}_{K},\left\{\varphi_{j}\right\}\right)$.

Remark 1.1. In [S5], $(A, \iota)$ in the above definition is called a principal abelian variety. But, in this paper, we will consider principally polarized abelian varieties. In order to avoid confusion, we use the terminology of the above definition.

Proposition 1.1. ([S5], Section 7) The abelian variety $(A(M), \iota)$ is of $C M$ type $\left(\mathfrak{O}_{K},\left\{\varphi_{j}\right\}\right)$ if and only if $M$ is a fractional ideal of $K$.

Let us consider abelian varieties $(A, \iota)=(A(\mathfrak{a}), \iota)$ coming from a fractional ideal $\mathfrak{a}$ of $K$. Take $(A, \Theta, \iota)$, where $\Theta$ is a polarization given by the Riemann form of (1.1). We call $(A, \Theta, \iota)$ a polarized abelian variety of type $\left(K,\left\{\varphi_{j}\right\} ; \zeta, \mathfrak{a}\right)$, or of type $(\zeta, \mathfrak{a})$. Let $\mathfrak{C}_{0}(K)$ be the abelian group consisting of all pairs $\{b, \mathfrak{c}\}$, where $b \in K_{0}$ is totally positive and $\mathfrak{c} \in I_{K}$. Here, the multiplication is given by $\left\{b_{1}, \mathfrak{c}_{1}\right\}\left\{b_{2}, \mathfrak{c}_{2}\right\}=$ $\left\{b_{1} b_{2}, \mathfrak{c}_{1} \mathfrak{c}_{2}\right\}$. Set $\mathfrak{C}(K)=\mathfrak{C}_{0}(K) /\left\{\left\{x x^{\rho}, x \mathfrak{O}_{K}\right\} \mid x \in K^{\times}\right\}$. We denote the coset of $\{b, \mathfrak{c}\}$ by $(b, \mathfrak{c})$. Suppose $P_{1}=\left(A_{1}, \Theta_{1}, \iota_{1}\right)\left(P_{2}=\left(A_{2}, \Theta_{2}, \iota_{2}\right)\right.$, resp. $)$ be a polarized abelian variety of type $\left(K,\left\{\varphi_{j}\right\} ; \zeta_{1}, \mathfrak{a}_{1}\right)$ $\left(\left(K,\left\{\varphi_{j}\right\} ; \zeta_{2}, \mathfrak{a}_{2}\right)\right.$, resp.). Setting $(b, \mathfrak{c})=\left(\zeta_{1}^{-1} \zeta_{2}, \mathfrak{a}_{2}^{-1} \mathfrak{a}_{1}\right)$, we put $\left(P_{2}: P_{1}\right)=(b, \mathfrak{c})$. We remark that $\left(A_{1}, \Theta_{1}\right)$ and $\left(A_{2}, \Theta_{2}\right)$ are isomorphic if and only if $\left(P_{2}: P_{1}\right)=\left(1, \mathfrak{O}_{K}\right)$.

\section{$1.2 \quad$ Reflex}

Definition 1.2. Let $\left(K,\left\{\varphi_{j}\right\}\right)$ be a CM type and $(A, \iota)$ be a corresponding abelian variety. If $A$ is a simple abelian variety, we call $\left(K,\left\{\varphi_{j}\right\}\right)$ primitive.

Proposition 1.2. ([S5], Section 8) Let $\left(K ;\left\{\varphi_{1}, \cdots, \varphi_{n}\right\}\right)$ be a CM type. Let $L$ be a Galois extension of $\mathbb{Q}$ containing $K$. Set $S$ be the set of all the elements of $\operatorname{Gal}(L / \mathbb{Q})$ inducing some $\varphi_{j}$ on $K_{0}$. Set $S^{*}=\left\{\sigma^{-1} \mid \sigma \in S\right\}$ and $H^{*}=\left\{\gamma \in \operatorname{Gal}(L / \mathbb{Q}) \mid \gamma S^{*}=S^{*}\right\}$. There exists the subfield $K^{*}$ of $L$ corresponding to the subgroup $H^{*}$ of $\operatorname{Gal}(L / \mathbb{Q})$. Let $\left\{\psi_{k}\right\}$ be the set of all the embeddings $K^{*} \hookrightarrow \mathbb{C}$ coming from $S^{*}$. Then, $\left(K^{*},\left\{\psi_{k}\right\}\right)$ is a primitive CM type. Moreover, $\left(K^{*},\left\{\psi_{k}\right\}\right)$ is determined only by $\left(K,\left\{\varphi_{j}\right\}\right)$.

Definition 1.3. For a CM type $\left(K,\left\{\varphi_{j}\right\}\right)$, the primitive CM type $\left(K^{*} ;\left\{\psi_{k}\right\}\right)$ in Proposition 1.2 is called the reflex of $\left(K,\left\{\varphi_{j}\right\}\right)$. 
Example 1.1. (see [S5], 8.4) Let $K_{0}$ be a real quadratic field and $K$ be a totally imaginary quadratic extension of $K_{0}$. Namely, let $K$ be a $C M$ field of degree 4 . Take $\zeta \in K$ such that $-\zeta^{2} \in K_{0}$ is totally positive. We can take $\varphi_{1}, \varphi_{2}: K \hookrightarrow \mathbb{C}$ such that $\varphi_{1}=i d$ and $\varphi_{2}=\varphi$ satisfying $\operatorname{Im}\left(\zeta^{\varphi}\right)>0$. Then, $\left(K,\left\{\varphi_{1}, \varphi_{2}\right\}\right)$ is a CM type. We have the following three cases.

(i) Suppose $\operatorname{Gal}(K / \mathbb{Q}) \simeq(\mathbb{Z} / 2 \mathbb{Z})^{2}$. Letting the group $S$ in Proposition 1.2 be $S=\{i d, \sigma\}$, we have $\operatorname{Gal}(K / \mathbb{Q})=\{i d, \sigma, \rho, \sigma \rho\}$, where $\rho$ is the complex conjugation. In this case, the CM type $(K,\{i d, \sigma\})$ is not primitive. The reflex $K^{*}$ is an imaginary quadratic field.

(ii) Suppose $\operatorname{Gal}(K / \mathbb{Q}) \simeq(\mathbb{Z} / 4 \mathbb{Z})$. Letting the group $S$ in Proposition 1.2 be $S=\{i d, \sigma\}$, we have $\operatorname{Gal}(K / \mathbb{Q})=\left\{i d, \sigma, \sigma^{2}=\rho, \sigma^{3}\right\}$, where $\rho$ is the complex conjugation. In this case, the CM type $(K,\{1, \sigma\})$ is primitive. The reflex of $(K,\{i d, \sigma\})$ is given by $\left(K^{*},\left\{\psi_{k}\right\}\right)=(K,\{1, \sigma\})$.

(iii) Suppose $K$ is not normal over $\mathbb{Q}$. Letting $K_{0}=\mathbb{Q}(\sqrt{\Delta})(\Delta>0)$, we can assume that $-\xi^{2}=$ $x+y \sqrt{\Delta}(x, y \in \mathbb{Q})$ is totally positive. Set $\xi=\sqrt{-1} \sqrt{x+y \sqrt{\Delta}}$ and $\xi^{\varphi}=\sqrt{-1} \sqrt{x-y \sqrt{\Delta}}$. Since $K$ is not normal, $\xi^{\varphi} \notin K$. So, the Galois closure $L$ is given by $L=\mathbb{Q}\left(\xi, \xi^{\varphi}\right)$. Set $\sigma:\left(\xi, \xi^{\varphi}\right) \mapsto\left(\xi^{\varphi},-\xi\right)$ and $\tau:\left(\xi, \xi^{\varphi}\right) \mapsto\left(\xi^{\varphi}, \xi\right)$. We can show that the Galois group $\operatorname{Gal}(L / \mathbb{Q})$ is generated by $\sigma$ and $\tau$. Moreover, the CM type $(K,\{1, \varphi\})$ is primitive and its reflex is given by $\left(\mathbb{Q}\left(\xi+\xi^{\varphi}\right),\{1, \sigma \tau\}\right)$.

\subsection{The field of moduli of principally polarized abelian varieties}

Let $k_{1}$ and $k_{2}$ be number fields. Let $\sigma: k_{1} \rightarrow k_{2}$ be an embedding. For an algebro-geometric object $W$ over $k_{1}$, we denote by $W^{\sigma}$ the image $W$ under $\sigma$. Then, $W^{\sigma}$ is defined over $k_{2}$.

Let $k_{1}$ be an algebraic number fields with $\operatorname{char}\left(k_{1}\right)=0$. Let $(A, \Theta)$ be a polarized abelian variety defined over $k_{1}$. Take an Galois extension $L$ of $k_{1}$ over $\mathbb{Q}$. We take a subgroup $G_{1}=\{\sigma \in$ $\operatorname{Gal}(L / \mathbb{Q}) \mid(A, \Theta)$ is isomorphic to $\left.\left(A^{\sigma}, \Theta^{\sigma}\right)\right\}$. Let $M$ be the subfield of $L$ corresponding to the subgroup $G_{1}$. We note that the field $M$ is uniquely determined by $(A, \Theta)$ and independent of the choice of the field of definition $k_{1}$ of $(A, \Theta)$ and the Galois closure $L$.

Definition 1.4. The above field $M$ is called the field of moduli of $(A, \Theta)$.

Remark 1.2. (WW], see also [S5]) Let $\operatorname{Aut}(A, \Theta)$ be the group of automorphisms of a polarized abelian variety $(A, \Theta)$. The quotient variety $A / \operatorname{Aut}(A, \Theta)$ is called the Kummer variety of $(A, \Theta)$ in the sense of $W e i l[W]$. Let $M$ be the field of moduli of $(A, \Theta)$. Then, the field of definition of the Kummer variety of $(A, \Theta)$ contains $M$. Moreover, there exists a model $W$ of the Kummer variety of $(A, \Theta)$ such that the field of definition of $W$ coincides with $M$.

In the rest of this subsection, we survey the field of moduli of principally polarized abelian surfaces. Let $(A, \Theta)$ be a simple and principally polarized abelian surface. Then, there exists a curve $C$ of genus 2 such that $A$ is equal to the Jacobian variety $\operatorname{Jac}(C)$ of $C$. Suppose $C$ is given by $C: y^{2}=u_{0}\left(x-x_{1}\right)(x-$ $\left.x_{2}\right)\left(x-x_{3}\right)\left(x-x_{4}\right)\left(x-x_{5}\right)\left(x-x_{6}\right)$. Then, we obtain the Igusa-Clebsch invariants $I_{2}, I_{4}, I_{6}$ and $I_{10}$ of $C$ :

$$
\left\{\begin{array}{l}
I_{2}=u_{0}^{2} \sum(12)^{2}(34)^{2}(56)^{2}, \quad I_{4}=u_{0}^{4} \sum(12)^{2}(23)^{2}(31)^{2}(45)^{2}(56)^{2}(64)^{2}, \\
I_{6}=u_{0}^{6} \sum(12)^{2}(23)^{2}(31)^{2}(45)^{2}(56)^{2}(64)^{2}(14)^{2}(25)^{2}(36)^{2}, \quad I_{10}=u_{0}^{10} \prod_{i<j}(i j)^{2} .
\end{array}\right.
$$

Here, $(j k)$ indicates $\left(x_{j}-x_{k}\right)$.

Let $\mathcal{M}_{2}$ be the moduli space of curves of genus 2 . Let $\mathbb{P}(1: 2: 3: 5)=\left\{\left(\zeta_{1}: \zeta_{2}: \zeta_{3}: \zeta_{5}\right)\right\}$ be the weighted projective space. It is well-known that $\mathcal{M}_{2}=\mathbb{P}(1: 2: 3: 5)-\left\{\zeta_{5}=0\right\}$. In fact, $\left(I_{2}: I_{4}: I_{6}: I_{10}\right)$ gives a well-defined point of the moduli space $\mathcal{M}_{2}$. We note that the moduli space $\mathcal{M}_{2}$ is a Zariski open set of the moduli space $\mathcal{A}_{2}$ of principally polarized abelian surfaces $\left(\mathcal{M}_{2}\right.$ is the complement of the divisor given by the points corresponding to the product of elliptic curves).

In [I] Igusa defined arithmetic invariants $J_{2}, J_{4}, J_{6}$ and $J_{10}$ given by

$$
J_{2}=2^{-3} I_{2}, \quad J_{10}=2^{-12} I_{10}, \quad J_{4}=2^{-5} 3^{-1}\left(4 J_{2}^{2}-I_{4}\right), \quad J_{6}=2^{-6} 3^{-2}\left(8 J_{2}^{3}-160 J_{2} J_{4}-I_{6}\right) .
$$


Due to (1.4), we have an isomorphism between the ring $\mathbb{Q}\left[I_{2}, I_{4}, I_{6}, I_{10}\right]$ and the ring $\mathbb{Q}\left[J_{2}, J_{4}, J_{6}, J_{10}\right]$.

For a curve $C$ of genus 2 , set

$$
m_{1}(C)=\frac{J_{2}^{5}}{J_{10}}, \quad m_{2}(C)=\frac{J_{2}^{3} J_{4}}{J_{10}}, \quad m_{3}(C)=\frac{J_{2}^{2} J_{6}}{J_{10}} .
$$

They are called the absolute invariants. Then, we have the following famous result (see [S2] or [FG]).

Proposition 1.3. (1) Let $C_{1}$ and $C_{2}$ be two curves of genus 2 with $I_{10} \neq 0$. Then, the curve $C_{1}$ is isomorphic to $C_{2}$ if and only if $m_{j}\left(C_{1}\right)=m_{j}\left(C_{2}\right)(j=1,2,3)$.

(2) Let $(A, \Theta)$ be a principally polarized abelian surface and $C$ be a curve of genus 2 such that $\operatorname{Jac}(C)=$ $A$. Then, a subfield $M$ of the field of definition of $(A, \Theta)$ gives a field of moduli of $(A, \Theta)$ if and only if the absolute invariants $m_{j}(C)(j=1,2,3)$ generate $M$ over $\mathbb{Q}$.

\subsection{Class fields from abelian varieties}

In this subsection, we give a survey of class fields derived from abelian varieties.

Take a cycle $\mathfrak{m}$ of $k$. Let $I_{k}(\mathfrak{m})$ be the group of fractional ideals of $k$ which are prime to $\mathfrak{m}$. Set $P_{k}(\mathfrak{m})=\{(\alpha) \mid \alpha \equiv 1(\bmod \mathfrak{m})\}$. Let $k_{1} / k$ be a Galois extension. Let $I_{k_{1}}(\mathfrak{m})$ be the group of fractional ideals of $k_{1}$ which are prime to $\mathfrak{m}$. The image $N_{k_{1} / k}\left(I_{k_{1}}(\mathfrak{m})\right)$ is a subgroup of $I_{k}(\mathfrak{m})$. Then, we have the fundamental inequality $\left[I_{k}(\mathfrak{m}): P_{k}(\mathfrak{m}) N_{k_{1} / k}\left(I_{k_{1}}(\mathfrak{m})\right)\right] \leq\left[k_{1}: k\right]$.

Definition 1.5. Let $H(\mathfrak{m})$ be a subgroup of $I_{k}(\mathfrak{m})$ such that $P_{k}(\mathfrak{m}) \subset H(\mathfrak{m}) \subset I_{k}(\mathfrak{m})$. If a Galois extension $k_{1} / k_{0}$ satisfies $\left[I_{k}(\mathfrak{m}): H(\mathfrak{m})\right]=\left[k_{1}: k\right]$ and $H(\mathfrak{m})=P_{k}(\mathfrak{m}) N_{k_{1} / k}\left(I_{k_{1}}(\mathfrak{m})\right)$, then $k_{1} / k$ is called the class field corresponding to $H(\mathfrak{m})$.

Under the notion in Definition 1.5, we have an isomorphism $I_{k}(\mathfrak{m}) / H(\mathfrak{m}) \simeq \operatorname{Gal}\left(k_{1} / k\right)$ that is given by the Artin symbol $\mathfrak{a} \mapsto\left(\frac{k_{1} / k}{\mathfrak{a}}\right)$ (The Artin reciprocity law). Let $\mathfrak{f}\left(k_{1} / k\right)$ be the conductor of $k_{1} / k$. If $\mathfrak{f}\left(k_{1} / k\right)=(1)$, then $k_{1} / k$ is unramified. Moreover, for any abelian extension $k_{1} / k$, there exists a group $H(\mathfrak{m})$ of Definition 1.5 such that $k_{1} / k$ is the class field corresponding to $H(\mathfrak{m})$.

Let $\left(K,\left\{\varphi_{j}\right\}\right)$ be a CM type and $\left(K^{*},\left\{\psi_{k}\right\}\right)$ be the corresponding reflex. Let $P=(A, \Theta, \iota)$ be a polarized abelian variety of type $\left(K,\left\{\varphi_{j}\right\}\right)$. Let $L$ be the Galois closure of $K$ over $\mathbb{Q}$. For $\mathfrak{a} \in I_{K^{*}}$, setting

$$
\mathfrak{a}^{\Phi^{*}}=\prod_{k} \mathfrak{a}^{\psi_{k}} .
$$

We can take $g(\mathfrak{a}) \in I_{K}$ such that $\mathfrak{O}_{L} g(\mathfrak{a})=\mathfrak{O}_{L} \mathfrak{a}^{\Phi^{*}}$. We need the subgroup $H_{0}$ of $I_{K^{*}}$ given by

$$
H_{0}=\left\{\mathfrak{a} \in I_{K^{*}} \mid g(\mathfrak{a}) \in P_{K}, \text { there exists } \mu \in K \text { such that } g(\mathfrak{a})=(\mu), N(\mathfrak{a})=\mu \mu^{\rho}\right\} .
$$

For $\sigma \in \operatorname{Gal}\left(\mathbb{C} / K^{*}\right), P^{\sigma}=\left(A^{\sigma}, \Theta^{\sigma}, \iota^{\sigma}\right)$ has the same type of $\left(K,\left\{\varphi_{j}\right\}\right)$. So, we obtain $\left(P^{\sigma}: P\right)=(b, \mathfrak{c})$ for some totally positive $b \in K_{0}$ and $\mathfrak{c} \in I_{K}$. So, we have a homomorphism $h: \operatorname{Gal}\left(\mathbb{C} / K^{*}\right) \rightarrow \mathfrak{C}(K)$ given by $\sigma \mapsto(b, \mathfrak{c}) . P$ is isomorphic to $P^{\sigma}$ if and only if $\sigma \in \operatorname{Ker}(h)$. Letting $M$ be the field of moduli of $(A, \Theta), \operatorname{Ker}(h)$ is the set of $\sigma$ which leaves the elements of $k_{0}=M K^{*}$ invariant. So, the homomorphism $h$ induces an embedding $\tilde{h}: \operatorname{Gal}\left(k_{0} / K^{*}\right) \hookrightarrow \mathfrak{C}(K)$. Especially, $k_{0} / K^{*}$ is an abelian extension. Hence, we can take an integral ideal $\mathfrak{m}_{0}$ of $K^{*}$ such that the Artin symbol $\sigma: I_{K^{*}}\left(\mathfrak{m}_{0}\right) \rightarrow \operatorname{Gal}\left(k_{0} / K^{*}\right)$ given by $\mathfrak{a} \mapsto \sigma(\mathfrak{a})=\left(\frac{k_{0} / K^{*}}{\mathfrak{a}}\right)$ induces the homomorphism $\tilde{h} \circ \sigma: I_{K^{*}}\left(\mathfrak{m}_{0}\right) \rightarrow \mathfrak{C}(K)$ with the form $\mathfrak{a} \mapsto$ $\left(P^{\sigma(\mathfrak{a})}: P\right)=\left(N(\mathfrak{a}), \mathfrak{a}^{\Phi^{*}}\right)$. By the definition (1.7), we can see that $\operatorname{Ker}(\sigma)=H_{0} \cap I_{K^{*}}\left(\mathfrak{m}_{0}\right)$. So, the Artin reciprocity law implies $I_{K^{*}}\left(\mathfrak{m}_{0}\right) /\left(H_{0} \cap I_{K^{*}}\left(\mathfrak{m}_{0}\right)\right) \simeq \operatorname{Gal}\left(k_{0} / K^{*}\right)$. Moreover, we can prove that $H_{0} \supset P_{K^{*}}=P_{K^{*}}((1))$ and the conductor $\mathfrak{f}\left(k_{0} / K^{*}\right)$ is (1). Thus, the following theorem follows.

Theorem 1.1. ([S5], Section 15) Let $\left(K,\left\{\varphi_{j}\right\}\right)$ be a $C M$ type and $\left(K^{*},\left\{\psi_{\alpha}\right\}\right)$ be the corresponding reflex. Let $H_{0}$ be the subgroup of $I_{K^{*}}$ given in 1.7). For a polarized abelian variety $P=(A, \Theta, \iota)$ of type $\left(K,\left\{\varphi_{j}\right\} ; \zeta, \mathfrak{a}\right)$ coming from $\zeta \in K$ and $\mathfrak{a} \in I_{K^{*}}$, let $M$ be the field of moduli of $(A, \Theta)$. Then, $k_{0}=M K^{*}$ is the unramified class field over $K^{*}$ corresponding to $H_{0}$. 


\section{Applications of $X, Y$ functions to class field theory}

We study the class fields in Section 1.4 for the case of $n=2$ and $K_{0}=\mathbb{Q}(\sqrt{5})$, that is the simplest case of complex multiplication of abelian surfaces because the discriminant of the real quadratic field is the smallest. In this section, we shall give an explicit construction of the class fields using the special values of the functions $X$ and $Y$ of (2.8).

\subsection{Klein's Icosahedral invariants and Hilbert modular surfaces}

Let $K_{0}=\mathbb{Q}(\sqrt{\Delta})$ be a real quadratic field with discriminant $\Delta$ and $\mathcal{O}_{\Delta}$ be the ring of integers. If an abelian surface $A$ and an embedding $\iota: K_{0} \hookrightarrow \operatorname{End}_{0}(A)$ satisfies $\iota\left(\mathcal{O}_{\Delta}\right) \subset \operatorname{End}(A)$, then the pair $(A, \iota)$ is called an abelian surface with real multiplication by $\mathcal{O}_{\Delta}$. The moduli space of principally polarized abelian surfaces with real multiplication by $\mathcal{O}_{\Delta}$ is called the Humbert surface $\mathcal{H}_{\Delta}$. The Humbert surface $\mathcal{H}_{\Delta}$ is a divisor in $\mathcal{A}_{2}$.

In our study, we focus on the case for the smallest discriminant $\Delta=5$. In this case, the corresponding ring of integers is $\mathcal{O}_{5}=\left\langle 1, \frac{1+\sqrt{5}}{2}\right\rangle_{\mathbb{Z}}$. The Humbert surface $\mathcal{H}_{5}$ is isomorphic to the symmetric Hilbert modular surface $\left\langle P S L\left(2, \mathcal{O}_{5}\right), \tau\right\rangle \backslash(\mathbb{H} \times \mathbb{H})$, where $\tau$ is the involution on $\mathbb{H} \times \mathbb{H}$ given by $\left(z_{1}, z_{2}\right) \mapsto\left(z_{2}, z_{1}\right)$ (see $[\mathrm{Ge}]$ ).

Proposition 2.1. ([피] (1) A compactification $\overline{\left\langle P S L\left(2, \mathcal{O}_{5}\right), \tau\right\rangle \backslash(\mathbb{H} \times \mathbb{H})}$ is isomorphic to the weighted projective plane $\mathbb{P}(1: 3: 5)=\operatorname{Proj}(\mathbb{C}[\mathfrak{A}, \mathfrak{B}, \mathfrak{C}])$, where $\mathfrak{A}, \mathfrak{B}$ and $\mathfrak{C}$ are Klein's icosahedral invariants.

(2) The Hilbert modular surface $\overline{P S L\left(2, \mathcal{O}_{5}\right) \backslash(\mathbb{H} \times \mathbb{H})}$ is isomorphic to the hypersurface $\operatorname{V}$ of $\mathbb{P}(2: 5$ : $6: 15)=\operatorname{Proj}(\mathbb{C}[\mathfrak{A}, \mathfrak{c}, \mathfrak{B}, \mathfrak{D}])$ defined by the equation

$$
144 \mathfrak{D}^{2}=-1728 \mathfrak{B}^{5}+720 \mathfrak{A c}^{2} \mathfrak{B}^{3}-80 \mathfrak{A}^{2} \mathfrak{c}^{4} \mathfrak{B}+64 \mathfrak{A}^{3}\left(5 \mathfrak{B}^{2}-\mathfrak{A c}^{2}\right)^{2}+\mathfrak{c}^{6} .
$$

Remark 2.1. In the above theorem, the Hilbert modular surface $\overline{P S L\left(2, \mathcal{O}_{5}\right) \backslash(\mathbb{H} \times \mathbb{H})} \simeq V$ is the double covering $\mathcal{R}$ of $\overline{\left\langle P S L\left(2, \mathcal{O}_{5}\right), \tau\right\rangle \backslash(\mathbb{H} \times \mathbb{H})} \simeq \mathbb{P}(1: 3: 5)=\operatorname{Proj}(\mathbb{C}[\mathfrak{A}, \mathfrak{B}, \mathfrak{C}])$ corresponding to the involution T. For any $(\mathfrak{A}: \mathfrak{B}: \mathfrak{C})$, we have $\mathcal{R}^{-1}(\mathfrak{A}: \mathfrak{B}: \mathfrak{C})=\{(\mathfrak{A}: \mathfrak{c}: \mathfrak{B}: \mathfrak{D}),(\mathfrak{A}:-\mathfrak{c}: \mathfrak{B}: \mathfrak{D})\} \subset V$, where $\mathfrak{c}^{2}=\mathfrak{C}$.

\subsection{The family $\mathcal{F}$ of $K 3$ surfaces and the period mapping}

In [N1], the family $\mathcal{F}=\{S(\mathfrak{A}: \mathfrak{B}: \mathfrak{C})\}$ of $K 3$ surfaces, where

$$
S(\mathfrak{A}: \mathfrak{B}: \mathfrak{C}): z^{2}=x^{3}-4\left(4 y^{3}-5 \mathfrak{A} y^{2}\right) x^{2}+20 \mathfrak{B} y^{3} x+\mathfrak{C} y^{4}
$$

is studied in detail. Here, $(\mathfrak{A}: \mathfrak{B}: \mathfrak{C}) \in \mathbb{P}(1: 3: 5)-\{(1: 0: 0)\}$. We note that we have an isomorphism $S(\mathfrak{A}: \mathfrak{B}: \mathfrak{C}) \mapsto S\left(\kappa^{2} \mathfrak{A}: \kappa^{6} \mathfrak{B}: \kappa^{10} \mathfrak{C}\right)$ of elliptic $K 3$ surfaces given by $(x, y, z) \mapsto\left(\kappa^{6} x, \kappa^{2} y, \kappa^{9} z\right)$ for $\kappa \neq 0$.

The multivalued period mapping $\Phi$ for $\mathcal{F}$ has the form $\Phi: \mathbb{P}(1: 3: 5)-\{(1: 0: 0)\} \rightarrow \mathbb{H} \times \mathbb{H}$ given by

$$
\left(z_{1}, z_{2}\right)=\left(-\frac{\int_{\Gamma_{3}} \omega+\frac{1-\sqrt{5}}{2} \int_{\Gamma_{4}} \omega}{\int_{\Gamma_{2}} \omega},-\frac{\int_{\Gamma_{3}} \omega+\frac{1+\sqrt{5}}{2} \int_{\Gamma_{4}} \omega}{\int_{\Gamma_{2}} \omega}\right),
$$

where $\omega$ is the unique holomorphic 2-form up to a constant factor and $\Gamma_{1}, \cdots, \Gamma_{4}$ are certain 2-cycles on $S(\mathfrak{A}: \mathfrak{B}: \mathfrak{C})$. In $[\mathbf{N 2}$, the above integrals are explicitly given by double integrals on a weighted projective plane. The inverse correspondence of $\Phi$ has an explicit expression by Hilbert modular functions as follows (for detail, see [N1]). Let us consider the holomorphic mapping $\mu_{5}: \mathbb{H} \times \mathbb{H} \rightarrow \mathfrak{S}_{2}$ given by

$$
\left(z_{1}, z_{2}\right) \mapsto \frac{1}{2 \sqrt{5}}\left(\begin{array}{cc}
(1+\sqrt{5}) z_{1}-(1-\sqrt{5}) z_{2} & 2\left(z_{1}-z_{2}\right) \\
2\left(z_{1}-z_{2}\right) & (-1+\sqrt{5}) z_{1}+(1+\sqrt{5}) z_{2}
\end{array}\right) .
$$

We note that $\mu_{5}$ of (2.4) gives a parametrization of the surface

$$
N_{5}=\left\{\left(\begin{array}{cc}
\tau_{1} & \tau_{2} \\
\tau_{2} & \tau_{3}
\end{array}\right) \in \mathfrak{S}_{2} \mid-\tau_{1}+\tau_{2}+\tau_{3}=0\right\}
$$


It is known that $\operatorname{pr}\left(N_{5}\right)$, where $p r: \mathfrak{S}_{2} \rightarrow S p(2, \mathbb{Z}) \backslash \mathfrak{S}_{2}=\mathcal{A}_{2}$ is the canonical projection, coincides with the Humbert surface $\mathcal{H}_{5}$. For $\Omega \in \mathfrak{S}_{2}$ and $a, b \in\{0,1\}^{2}$ with ${ }^{t} a b \equiv 0(\bmod 2)$, set $\vartheta(\Omega ; a, b)=$ $\sum_{g \in \mathbb{Z}^{2}} \exp \left(\pi \sqrt{-1}\left({ }^{t}\left(g+\frac{1}{2} a\right) \Omega\left(g+\frac{1}{2} a\right)+{ }^{t} g b\right)\right)$. For $j \in\{0,1, \cdots, 9\}$, we set $\theta_{j}\left(z_{1}, z_{2}\right)=\vartheta\left(\mu_{5}\left(z_{1}, z_{2}\right) ; a, b\right)$, where the correspondence between $j$ and $(a, b)$ is given by Table 2 . Let $a \in \mathbb{Z}$ and $j_{1}, \cdots, j_{r} \in\{0, \cdots, 9\}$.

\begin{tabular}{ccccccccccc}
\hline$j$ & 0 & 1 & 2 & 3 & 4 & 5 & 6 & 7 & 8 & 9 \\
\hline${ }^{t} a$ & $(0,0)$ & $(1,1)$ & $(0,0)$ & $(1,1)$ & $(0,1)$ & $(1,0)$ & $(0,0)$ & $(1,0)$ & $(0,0)$ & $(0,1)$ \\
${ }^{t} b$ & $(0,0)$ & $(0,0)$ & $(1,1)$ & $(1,1)$ & $(0,0)$ & $(0,0)$ & $(0,1)$ & $(0,1)$ & $(1,0)$ & $(1,0)$ \\
\hline
\end{tabular}

Table 2: The correspondence between $j$ and $(a, b)$.

We set $\theta_{j_{1}, \cdots, j_{r}}^{a}=\theta_{j_{1}}^{a} \cdots \theta_{j_{r}}^{a}$. The following $g_{2}\left(s_{5}, s_{6}, s_{15}\right.$, resp. $)$ is a symmetric Hilbert modular form of weight $2(5,6,15$, resp.) for $\mathbb{Q}(\sqrt{5})$ (see Müller [Mul]):

$$
\left\{\begin{array}{l}
g_{2}=\theta_{0145}-\theta_{1279}-\theta_{3478}+\theta_{0268}+\theta_{3569}, \quad s_{5}=2^{-6} \theta_{0123456789} \\
s_{6}=2^{-8}\left(\theta_{012478}^{2}+\theta_{012569}^{2}+\theta_{034568}^{2}+\theta_{236789}^{2}+\theta_{134579}^{2}\right) \\
s_{15}=-2^{-18}\left(\theta_{07}^{9} \theta_{18}^{5} \theta_{24}-\theta_{25}^{9} \theta_{16}^{5} \theta_{09}+\theta_{58}^{9} \theta_{03}^{5} \theta_{46}-\theta_{09}^{9} \theta_{25}^{5} \theta_{16}+\theta_{09}^{9} \theta_{16}^{5} \theta_{25}-\theta_{67}^{9} \theta_{23}^{5} \theta_{89}\right. \\
+\theta_{18}^{9} \theta_{24}^{5} \theta_{07}-\theta_{24}^{9} \theta_{18}^{5} \theta_{07}-\theta_{46}^{9} \theta_{03}^{5} \theta_{58}-\theta_{24}^{9} \theta_{07}^{5} \theta_{18}-\theta_{89}^{9} \theta_{67}^{5} \theta_{23}-\theta_{07}^{9} \theta_{24}^{5} \theta_{18} \\
+\theta_{89}^{9} \theta_{23}^{5} \theta_{67}-\theta_{49}^{9} \theta_{13}^{5} \theta_{57}+\theta_{16}^{9} \theta_{09}^{5} \theta_{25}-\theta_{03}^{9} \theta_{46}^{5} \theta_{58}+\theta_{16}^{9} \theta_{25}^{5} \theta_{09}-\theta_{46}^{9} \theta_{58}^{5} \theta_{03} \\
\quad-\theta_{25}^{9} \theta_{09}^{5} \theta_{16}-\theta_{57}^{9} \theta_{49}^{5} \theta_{13}+\theta_{67}^{9} \theta_{89}^{5} \theta_{23}+\theta_{58}^{9} \theta_{46}^{5} \theta_{03}+\theta_{57}^{9} \theta_{13}^{5} \theta_{49}-\theta_{23}^{9} \theta_{89}^{5} \theta_{67} \\
\left.+\theta_{18}^{9} \theta_{07}^{5} \theta_{24}+\theta_{03}^{9} \theta_{58}^{5} \theta_{46}+\theta_{23}^{9} \theta_{67}^{5} \theta_{89}+\theta_{49}^{9} \theta_{57}^{5} \theta_{13}-\theta_{13}^{9} \theta_{57}^{5} \theta_{49}+\theta_{13}^{9} \theta_{49}^{5} \theta_{57}\right) .
\end{array}\right.
$$

Proposition 2.2. (N1] Theorem 4.1) The inverse correspondence $\mathbb{H} \times \mathbb{H} \rightarrow \mathbb{P}(1: 3: 5)$ of the multivalued period mapping $\Phi$ is given by

$$
\left(z_{1}, z_{2}\right) \mapsto(\mathfrak{A}: \mathfrak{B}: \mathfrak{C})=\left(g_{2}\left(z_{1}, z_{2}\right): 2^{5} \cdot 5^{2} s_{6}\left(z_{1}, z_{2}\right): 2^{10} \cdot 5^{5} s_{10}\left(z_{1}, z_{2}\right)\right) .
$$

Setting $X=\frac{\mathfrak{B}}{\mathfrak{A}^{3}}, Y=\frac{\mathfrak{C}}{\mathfrak{A}^{5}},(X, Y)$ gives a system of affine coordinates of $\mathbb{P}(1: 3: 5)$. From (2.7), we have the meromorphic functions

$$
X\left(z_{1}, z_{2}\right)=2^{5} \cdot 5^{2} \cdot \frac{s_{6}\left(z_{1}, z_{2}\right)}{g_{2}^{3}\left(z_{1}, z_{2}\right)}, \quad Y\left(z_{1}, z_{2}\right)=2^{10} \cdot 5^{5} \cdot \frac{s_{10}\left(z_{1}, z_{2}\right)}{g_{2}^{5}\left(z_{1}, z_{2}\right)}
$$

on $\mathbb{H} \times \mathbb{H}$. We call them $X, Y$ functions.

\subsection{The Shioda-Inose structure of $\mathcal{F}$}

Let $A$ be an abelian variety. The minimal resolution $\operatorname{Kum}(A)$ of the quotient variety $A /\left\{i d_{A},-i d_{A}\right\}$ is called the $\operatorname{Kummer}$ surface. We note that $\operatorname{Kum}(A)$ is an algebraic $K 3$ surface.

Let $S$ be an algebraic $K 3$ surface. Let $\omega$ be the unique holomorphic 2-form on $S$ up to a constant factor. If an involution $\nu$ on $S$ satisfies $\nu^{*} \omega=\omega$, we call $\nu$ a symplectic involution. Set $G=\{i d, \nu\} \subset \operatorname{Aut}(S)$. The minimal resolution $S^{\prime}$ of the quotient variety $S / G$ is a $K 3$ surface. We have the rational quotient mapping $\chi: S \rightarrow S^{\prime}$. We say that a $K 3$ surface $S$ admits a Shioda-Inose structure if there exists a symplectic involution $\iota \in \operatorname{Aut}(S)$ with the rational quotient mapping $\chi: S \rightarrow S^{\prime}$ such that $S^{\prime}$ is a Kummer surface and $\chi_{*}$ induces a Hodge isometry $\operatorname{Tr}(S)(2) \simeq \operatorname{Tr}\left(S^{\prime}\right)$.

Proposition 2.3. (N2] Section 2) If a principally polarized abelian surface $(A, \Theta)$ has real multiplication by $\mathcal{O}_{5}$, then there exists a Hodge isometry

$$
\operatorname{Tr}(A) \simeq \operatorname{Tr}(S(\mathfrak{A}: \mathfrak{B}: \mathfrak{C}))
$$

for some $(\mathfrak{A}: \mathfrak{B}: \mathfrak{C}) \in \mathbb{P}(1: 3: 5)-\{(1: 0: 0)\}$ via the Shioda-Inose structure of $S(\mathfrak{A}: \mathfrak{B}: \mathfrak{C})$.

Remark 2.2. The corresponding Kummer surface $K(X, Y)$ coming from the Shioda-Inose structure with $\chi: S(\mathfrak{A}: \mathfrak{B}: \mathfrak{C}) \rightarrow K(X, Y)$ is given by the elliptic surface

$$
K(X, Y): v^{2}=\left(u^{2}-2 y^{5}\right)\left(u-\left(5 y^{2}-10 X y+Y\right)\right) .
$$




\subsection{The field of moduli via periods of $K 3$ surfaces}

Let $\mathfrak{S}_{2}$ be the Siegel upper half plane of degree 2. Let $S p(2, \mathbb{Z})$ be the symplectic group consisting of $4 \times 4$ matrices. It is well known that the Igusa 3 -fold $\mathcal{A}_{2}$ of principally polarized abelian surfaces is given by the quotient space $S p(2, \mathbb{Z}) \backslash \mathfrak{S}_{2}$. Here, recall that the moduli space $\mathcal{M}_{2}=\operatorname{Proj}\left(\mathbb{C}\left[I_{2}, I_{4}, I_{6}, I_{10}\right]\right)$ of genus 2 curves is a Zariski open set of $\mathcal{A}_{2}$.

Proposition 2.4. Let $(A, \Theta)$ be a principally polarized abelian surface with real multiplication by $\mathcal{O}_{5}$. Let $S(\mathfrak{A}: \mathfrak{B}: \mathfrak{C})$ be the corresponding K3 surface under the Hodge isometry of (2.9). Then, the field of moduli of $(A, \Theta)$ is given by $\mathbb{Q}(X, Y)$.

Proof. We note that, by Proposition 1.3 , the field of moduli $(A, \Theta)$ is given by $\mathbb{Q}\left(m_{1}, m_{2}, m_{3}\right)$.

Let us introduce the family $\mathcal{F}_{C D}=\left\{S_{C D}(\alpha: \beta: \gamma: \delta)\right\}$ of $K 3$ surfaces, where $(\alpha, \beta, \gamma, \delta) \in \mathbb{P}(2: 3:$ $5: 6)-\{\gamma=\delta=0\}$. The member $S_{C D}(\alpha: \beta: \gamma: \delta)$ is defined by the explicit equation

$$
S_{C D}(\alpha: \beta: \gamma: \delta): y^{2}=x^{3}+\left(-3 \alpha t^{4}-\gamma t^{5}\right) x+\left(t^{5}-2 \beta t^{6}+\delta t^{7}\right)
$$

of an elliptic $K 3$ surface. This $K 3$ surface is due to Kumar [K] or Clingher and Doran [CD]. Here, the moduli space for $\mathcal{F}_{C D}$ is isomorphic to the Igusa 3-fold $\mathcal{A}_{2}=S p(2, \mathbb{Z}) \backslash \mathfrak{S}_{2}$. Then, $\left(I_{2}: I_{4}: I_{6}: I_{10}\right) \in$ $\mathbb{P}(1: 2: 3: 5)-\left\{I_{10}=0\right\}$ corresponds to a point $(\alpha: \beta: \gamma: \delta) \in \mathbb{P}(2: 3: 5: 6)-\{\gamma=\delta=0\}$, via the period mapping of $\mathcal{F}_{C D}$. Namely, we have the following mapping over $\mathbb{Q}$ :

$$
\alpha=\frac{1}{9} I_{4}, \quad \beta=\frac{1}{27}\left(-I_{2} I_{4}+3 I_{6}\right), \quad \gamma=8 I_{10}, \quad \delta=\frac{2}{3} I_{2} I_{10} .
$$

Moreover, $S_{C D}(\alpha: \beta: \gamma: \delta)$ has the Shioda-Inose structure and there exists the Hodge isometry

$$
\operatorname{Tr}(A) \simeq \operatorname{Tr}\left(S_{C D}(\alpha: \beta: \gamma: \delta)\right)
$$

where $A$ is a generic principally polarized abelian surface. Under (2.13), an principally polarized abelian surface corresponds to $S(\alpha: \beta: \gamma: \delta)$ (see [CD] or [NS1]).

In N3 Theorem 3.16, we proved that an principally polarized abelian surface $A$ has real multiplication by $\mathcal{O}_{5}$ if and only if $(\alpha: \beta: \gamma: \delta)$ is a point of $\Psi_{5}(\mathbb{P}(1: 3: 5))$, where $\Psi_{5}: \mathbb{P}(1: 3: 5) \rightarrow \mathbb{P}(2: 3: 5: 6)$ is given by

$$
\left\{\begin{array}{l}
\alpha_{5}(\mathfrak{A}: \mathfrak{B}: \mathfrak{C})=\frac{25}{36} \mathfrak{A}^{2}, \quad \beta_{5}(\mathfrak{A}: \mathfrak{B}: \mathfrak{C})=\frac{1}{2}\left(-\frac{125}{108} \mathfrak{A}^{3}+\frac{5}{4} \mathfrak{B}\right) \\
\gamma_{5}(\mathfrak{A}: \mathfrak{B}: \mathfrak{C})=\frac{1}{32} \mathfrak{C}, \quad \delta_{5}(\mathfrak{A}: \mathfrak{B}: \mathfrak{C})=\frac{25}{64} \mathfrak{B}^{2}-\frac{5}{96} \mathfrak{A C}
\end{array}\right.
$$

Especially, $\Psi_{5}$ is defined over $\mathbb{Q}$.

From (2.12) and (1.4), we have

$$
\mathbb{Q}\left[J_{2}, J_{4}, J_{6}, J_{10}\right]=\mathbb{Q}[\alpha, \beta, \gamma, \delta] .
$$

Moreover, if a principally polarized abelian surface $(A, \Theta)$ has real multiplication by $\mathcal{O}_{5}$, by virtue of the embedding $\Psi_{5}$ of (2.14), we have

$$
\mathbb{Q}[\alpha, \beta, \gamma, \delta]=\mathbb{Q}[\mathfrak{A}, \mathfrak{B}, \mathfrak{C}] .
$$

Hence, from (1.5), (2.15) and (2.16), if $(A, \Theta)$ has real multiplication by $\mathcal{O}_{5}$, we have $\mathbb{Q}\left(m_{1}, m_{2}, m_{3}\right)=$ $\mathbb{Q}(X, Y)$.

Remark 2.3. Due to Proposition 2.4, the equation (2.10) of the Kummer surface gives an explicit model of the Kummer variety $W$ in the sense of Remark 1.2. To see this, we note that the torsion part $E_{\operatorname{End}(A)}^{\text {tor }}$ of the group $E_{\operatorname{End}(A)}$ of all the units of $\operatorname{End}(A)$ contains $\operatorname{Aut}(A, \Theta)$ (see [S5]). If a unit $u$ in the ring $\mathcal{O}_{5}$ is a torsion element, then $u=1$ or $u=-1$. This implies that $\operatorname{Aut}(A, \Theta)=\left\{i d_{A},-i d_{A}\right\}$. 
Let us apply the argument in Section 1.1 to our case. Let $K$ be an imaginary quadratic extension over $K_{0}=\mathbb{Q}(\sqrt{5})$. Let $(K,\{i d, \sigma\})$ be a CM type of $K$. We set $u(\alpha)=\left(\begin{array}{c}\alpha \\ \alpha^{\sigma}\end{array}\right) \in \mathbb{C}^{2}$ for $\alpha \in K$. Take $\mathfrak{a} \in I_{K}$. For a system $\left\{\alpha_{1}, \cdots, \alpha_{4}\right\}$ of the basis of $\mathfrak{a}$, the $2 \times 4$ matrix $\left(u\left(\alpha_{1}\right) \cdots u\left(\alpha_{4}\right)\right)$ gives a lattice $\Lambda(\mathfrak{a})$ and we have a complex torus $\mathbb{C}^{2} / \Lambda(\mathfrak{a})=A(\mathfrak{a})$. If the alternating form $E$ of (1.1) given by $\zeta \in K$ gives a polarization $\Theta$ on $A(\mathfrak{a}),(A(\mathfrak{a}), \Theta)$ is a polarized abelian surface and $(A(\mathfrak{a}), \iota)$ is of CM type $\left(\mathfrak{D}_{K},\left\{\varphi_{j}\right\}\right)$ in the sense of Definition 1.1 (see Proposition 1.1).

Proposition 2.5. In the above notation, suppose $\Theta$ is a principal polarization.

(1) There exists a basis of $\mathbb{C}^{2}$ such that the matrix $\left(u\left(\alpha_{1}\right) \cdots u\left(\alpha_{4}\right)\right)$ is expressed in the form

$$
\left(u\left(\alpha_{1}\right) u\left(\alpha_{2}\right) u\left(\alpha_{3}\right) u\left(\alpha_{4}\right)\right)=\left(\begin{array}{cccc}
\tau_{1} & \tau_{2} & 1 & 0 \\
\tau_{2} & \tau_{3} & 0 & 1
\end{array}\right) \quad\left(\left(\begin{array}{ll}
\tau_{1} & \tau_{2} \\
\tau_{2} & \tau_{3}
\end{array}\right) \in \mathfrak{S}_{2}, \quad \tau_{1}=\tau_{2}+\tau_{3}\right) .
$$

(2) Put

$$
z_{1}^{0}=\frac{\tau_{2}+\sqrt{5} \tau_{2}+2 \tau_{3}}{2}, \quad z_{2}^{0}=\frac{\tau_{2}-\sqrt{5} \tau_{2}+2 \tau_{3}}{2} .
$$

Then, the field of moduli of $(A, \Theta)$ is given by $\mathbb{Q}\left(X\left(z_{1}^{0}, z_{2}^{0}\right), Y\left(z_{1}^{0}, z_{2}^{0}\right)\right)$, where

$$
X\left(z_{1}^{0}, z_{2}^{0}\right)=2^{5} \cdot 5^{2} \cdot \frac{s_{6}\left(z_{1}^{0}, z_{2}^{0}\right)}{g_{2}^{3}\left(z_{1}^{0}, z_{2}^{0}\right)}, \quad Y\left(z_{1}^{0}, z_{2}^{0}\right)=2^{10} \cdot 5^{5} \cdot \frac{s_{10}\left(z_{1}^{0}, z_{2}^{0}\right)}{g_{2}^{5}\left(z_{1}^{0}, z_{2}^{0}\right)} .
$$

Proof. (1) Under the assumption, $A$ satisfies $\mathfrak{O}_{K}=\operatorname{End}(A)$. So, $(A, \Theta)$ gives a principally polarized abelian surface with real multiplication by $\mathcal{O}_{5}$. Recall that the Humbert surface $\mathcal{H}_{5}$ is given by $\operatorname{pr}\left(N_{5}\right)$, where $N_{5}$ is given in (2.5). Then, the assertion follows.

(2) For a principally polarized abelian surface $(A, \Theta)$ with real multiplication by $\mathcal{O}_{5}$, there exists a $K 3$ surface $S(\mathfrak{A}: \mathfrak{B}: \mathfrak{C})$ via the correspondence (2.9). When $(A, \Theta)$ is given by the period matrix (2.17), due to Proposition 2.2, 2.3, 2.4 and the modular embedding (2.17), we have the assertion.

From Theorem 1.1 and the above proposition (2), the following theorem holds.

Theorem 2.1. In the above notation, let $K^{*}$ be the reflex of the CM type $(K,\{i d, \varphi\})$. Then, the field $K^{*}\left(X\left(z_{1}^{0}, z_{2}^{0}\right), Y\left(z_{1}^{0}, z_{2}^{0}\right)\right)$ is the unramified class field over $K^{*}$ for the group $H_{0}$ of (1.7).

Remark 2.4. Lauter and Yang $[L Y]$ introduced Gundlach invariants based on the work of Gundlach [Gl]. By combining the arguments of $[G \mathrm{Gl}],[M u l]$ and $[L Y]$, we can obtain an expression of the pair of our $X, Y$ functions in terms of Gundlach invariants. In fact, we can give another proof via Gundlach invariants of Theorem 2.1. The reason why we give the proof based on the periods of K3 surfaces is that our argument has the following good points.

- In our research, $X, Y$ functions are Hilbert modular functions of the coordinates $\left(z_{1}, z_{2}\right)$ of (2.3) given by the period of K3 surfaces. In Theorem [2.3 (the paper [N3], resp.), explicit models of Shimura varieties (Shimura curves, resp.) are studied, using $X, Y$ functions in the above sense.

- It is expected that the family $\left\{S_{C D}(\alpha: \beta: \gamma: \delta)\right\}$ of (2.11) are useful to construct class fields and explicit models of Shimura varieties in many other cases. The argument in this paper gives a prototype of such applications of $K 3$ surfaces to number theory.

Remark 2.5. K3 surfaces coming from toric varieties are important object in mirror symmetry. Our K3 surface $S(\mathfrak{A}: \mathfrak{B}: \mathfrak{C})$, in fact, is given by a hypersurface of a toric 3 -fold. In the study of mirror symmetry, toroidal compactifications of moduli spaces are very important. In the paper [HNU], a toroidal compactification from the viewpoint of mirror symmetry was given in terms of $X, Y$ functions. So, Theorem 2.1 gives an explicit example connecting number theory and mirror symmetry. 


\subsection{The canonical model of a Shimura variety}

Let $K_{0}$ be a totally real field of degree $n$ and $B$ be a quaternion algebra over $K_{0}$ such that $B \otimes_{K_{0}} \mathbb{R} \simeq$ $M_{2}(\mathbb{R})^{n}$. Let $\mathfrak{O}_{B}$ be a maximal order of $B$. We set $\Gamma_{1}\left(\mathfrak{O}_{B}\right)=\left\{\gamma \in \mathfrak{O}_{B} \mid \operatorname{Nr}_{B / K_{0}}(\gamma)=1\right\}$. Then, $\Gamma_{1}\left(\mathfrak{O}_{B}\right)$ acts on $\mathbb{H}^{n}$. Let $K$ be a totally imaginary quadratic extension over $K_{0}$ with an $K_{0}$-linear embedding $f: K \hookrightarrow B$. We can see that there exists the unique fixed point $z_{0} \in \mathbb{H}^{n}$ of $f(K) \subset B \otimes_{K_{0}} \mathbb{R} \simeq M_{2}(\mathbb{R})^{n}$ (see [S3 2.6). Shimura S3] studied such quotient spaces $\Gamma_{1}\left(\mathfrak{O}_{B}\right) \backslash \mathbb{H}^{n}$ deeply and defined the canonical models. They are the origin of Shimura varieties.

Theorem 2.2. ([S3] 9.3) In the above notation, there exist a Zariski open subset $V_{S}$ of a projective variety and a holomorphic mapping $\varphi_{S}: \mathbb{H}^{n} \rightarrow V_{S}$ satisfying the following conditions.

$(S-i) V_{S}$ is normal and defined over $\mathbb{Q}$.

(S-ii) $\varphi_{S}$ gives a biregular isomorphism $\Gamma_{1}\left(\mathfrak{O}_{B}\right) \backslash \mathbb{H}^{n} \simeq V_{S}$

(S-iii) Suppose $f$ satisfies $f\left(\mathfrak{O}_{K}\right) \subset \mathfrak{O}_{B}$. Then, $K^{*}\left(\varphi_{S}(z)\right) / K^{*}$ gives the class field for the group $H_{0}$ of (1.7).

The pair $\left(V_{S}, \varphi\right)$ in the above theorem is called the canonical model of $\Gamma_{1}\left(\mathfrak{O}_{B}\right) \backslash \mathbb{H}^{n}$.

If $K_{0}$ is a real quadratic field $\mathbb{Q}(\sqrt{\Delta})$ and $B=M_{2}(\mathbb{Q}(\sqrt{\Delta}))$, then $\mathfrak{O}_{B}=M_{2}\left(\mathcal{O}_{\Delta}\right)$ and $\Gamma_{1}\left(\mathfrak{O}_{B}\right) \backslash(\mathbb{H} \times \mathbb{H})$ coincides with the Hilbert modular surface $\operatorname{PSL}\left(2, \mathcal{O}_{\Delta}\right) \backslash(\mathbb{H} \times \mathbb{H})$. In this section, as an application of Theorem 2.1 we obtain the canonical model for the simplest case.

Theorem 2.3. Let $V$ be the variety of Proposition 2.1 (2). The holomorphic mapping $\varphi: \mathbb{H} \times \mathbb{H} \rightarrow V$ defined by

$$
\left(z_{1}, z_{2}\right) \mapsto(\mathfrak{A}: \mathfrak{c}: \mathfrak{B}: \mathfrak{D})=\left(g_{2}\left(z_{1}, z_{2}\right): 2^{5} \cdot 5^{2} \sqrt{5} s_{5}\left(z_{1}, z_{2}\right): 2^{5} \cdot 5^{2} g_{6}\left(z_{1}, z_{2}\right): 2^{13} \cdot 5^{5} \cdot 3^{-1} s_{15}\left(z_{1}, z_{2}\right)\right)
$$

gives the canonical model $(V, \varphi)$ of the $\Gamma_{1}\left(\mathfrak{O}_{B}\right) \backslash(\mathbb{H} \times \mathbb{H})$ for $K_{0}=\mathbb{Q}(\sqrt{5})$ and $B=M_{2}\left(K_{0}\right)$.

Proof. From Proposition 2.2 we can see that $(V, \varphi)$ of (2.20) satisfies $(S$-i) and $(S$-ii). Here, we note that the coefficient $2^{13} \cdot 5^{5} \cdot 3^{-1}$ of $s_{15}$ was calculated in the proof of [N1 Theorem 4.1. In this proof, we shall show that $(V, \varphi)$ satisfies $(S$-iii).

For the real quadratic field $\mathbb{Q}(\sqrt{\Delta})$ of discriminant $\Delta$, let us consider a principally polarized abelian variety $(A, \Theta)$ with $\tilde{\iota}: \mathbb{Q}(\sqrt{\Delta}) \hookrightarrow \operatorname{End}_{0}(A)$. We note that we have two choices of $\tilde{\iota}$ induced from the choices of the embeddings $\mathbb{Q}(\sqrt{\Delta}) \hookrightarrow \mathbb{R}$ given by $\sqrt{\Delta} \mapsto \sqrt{\Delta}$ or $\sqrt{\Delta} \mapsto-\sqrt{\Delta}$. For $\sigma \in \operatorname{Aut}(\mathbb{C})$, set $\tilde{\iota}^{\sigma}(a)=(\tilde{\iota}(a))^{\sigma}(a \in \mathbb{Q}(\sqrt{\Delta}))$. For a triplet $(A, \Theta, \tilde{\iota})$, we can consider $P^{\sigma}=\left(A^{\sigma}, \Theta^{\sigma}, \tilde{\iota}^{\sigma}\right)$ and define the field of moduli $M^{\prime}$ of $P=(A, \Theta, \tilde{\iota})$. Namely, $P^{\sigma}$ is isomorphic to $P$ if and only if $\sigma \in \operatorname{Gal}\left(\mathbb{C} / M^{\prime}\right)$. We note that the field of moduli $M^{\prime}$ of $(A, \Theta, \tilde{\iota})$ is an algebraic extension of the field of moduli $M$ of $(A, \Theta)$ given in Definition 1.4 (see [S1] Proposition 8).

Suppose that $\Delta$ is equal to a sum of two squares of prime numbers. Then, according to Ge Chapter IX Section 2, the moduli space of $(A, \Theta)$ is given by the Humbert surface, which is isomorphic to $\left\langle P S L\left(2, \mathcal{O}_{\Delta}\right), \tau\right\rangle \backslash(\mathbb{H} \times \mathbb{H})$, and the forgetting mapping $(A, \Theta, \tilde{\iota}) \mapsto(A, \Theta)$ corresponds to the action of the involution $\tau$ of $\mathbb{H} \times \mathbb{H}$ given by $\left(z_{1}, z_{2}\right) \mapsto\left(z_{2}, z_{1}\right)$.

From now on, we consider the case $\Delta=1^{2}+2^{2}$. By Proposition 2.5 (2), the field of moduli $M$ of $(A, \Theta)$ coincides with $\mathbb{Q}(X, Y)=\mathbb{Q}\left(\frac{\mathfrak{B}}{\mathfrak{A}^{3}}, \frac{\mathfrak{C}}{\mathfrak{A}^{5}}\right)$ under our construction. By virtue of Remark 2.1 and the argument in the above paragraph, the forgetting mapping $(A, \Theta, \tilde{\iota}) \mapsto(A, \Theta)$ is described by the double covering $\mathcal{R}: V \rightarrow \mathbb{P}(1: 3: 5)$ in Remark 2.1 On $(\mathfrak{A}: \mathfrak{B}: \mathfrak{C}) \in \mathbb{P}(1: 3: 5)$, there exist two points $(\mathfrak{A}: \pm \mathfrak{c}: \mathfrak{B}: \mathfrak{D}) \in V$ corresponding to the choices of $\tilde{\iota}$. So, the field of moduli $M^{\prime}$ of $(A, \Theta, \tilde{\iota})$ is given by $\mathbb{Q}(X, \eta)$, where $(X, \eta)=\left(\frac{\mathfrak{B}}{\mathfrak{A}^{3}}, \frac{\mathfrak{D}}{\mathfrak{c}^{3}}\right)$ is a system of affine coordinates of $V$.

Let $K$ be an imaginary quadratic extension of $K_{0}=\mathbb{Q}(\sqrt{5})$ and $\left(K,\left\{\varphi_{j}\right\}\right)$ is a CM type. We suppose that $(A, \Theta)$ is a principally polarized abelian surface of type $\left(K,\left\{\varphi_{j}\right\}\right)$. So, as in Section 1.1, we have the isomorphism $\iota: K \simeq \operatorname{End}_{0}(A)$, which induces $\tilde{\iota}=\left.\iota\right|_{\mathbb{Q}(\sqrt{5})}: K \hookrightarrow \operatorname{End}_{0}(A)$. In this case, we are able 
to apply [S5] Chapter IV Proposition 1. By this proposition, together with the definition of the field of moduli $M$ of $(A, \Theta)$, we have $(A, \Theta, \iota) \simeq\left(A^{\sigma} . \Theta^{\sigma}, \iota^{\sigma}\right)$ for any $\sigma \in \mathrm{Gal}(\mathbb{C} / M)$. Of course, this induces that $(A, \Theta, \tilde{\iota}) \simeq\left(A^{\sigma} . \Theta^{\sigma}, \tilde{\iota}^{\sigma}\right)$. Therefore, if $(A, \Theta, \tilde{\iota})$ is of CM type, the field of moduli $M^{\prime}$ of $(A, \Theta, \tilde{\iota})$ must coincide with the field of moduli $M$ of $(A, \Theta)$. Hence, in our case, we have

$$
\mathbb{Q}(X, Y)=\mathbb{Q}(X, \eta) .
$$

Here, we suppose that there is a $K_{0}$-linear embedding $f: K \hookrightarrow M_{2}\left(\mathfrak{O}_{B}\right)$ with $f\left(\mathfrak{O}_{K}\right) \subset \operatorname{End}(A)$ and $\left(z_{1}^{0}, z_{2}^{0}\right) \in \mathbb{H} \times \mathbb{H}$ is the unique fixed point of $f(K)$. By virtue of the argument [S3] 2.7, such an embedding $f$ determines a CM type $\left(K,\left\{\varphi_{j}\right\}\right)$. This CM type determines a pair $(A, \iota)$, where $\iota: K \hookrightarrow \operatorname{End}_{0}(A)$. Since $\left(z_{1}^{0}, z_{2}^{0}\right)$ is the fixed point for $f(K)$, where $f$ satisfies $f\left(\mathfrak{O}_{K}\right) \subset \operatorname{End}(A)$, by the argument of [Ge] Chapter IX Section 1, we can see that the abelian surface $A$ coming from the fixed point $\left(z_{1}^{0}, z_{2}^{0}\right)$ has a principal polarization $\Theta$ and the pair $(A, \iota)$ is of CM type $\left(\mathfrak{O}_{K},\left\{\varphi_{1}, \varphi_{2}\right\}\right)$ in the sense of Definition 1.1. So, by our construction via the Shioda-Inose structure of $\mathcal{F}=\{S(\mathfrak{A}: \mathfrak{B}: \mathfrak{C})\}, \mathbb{Q}\left(X\left(z_{1}^{0}, z_{2}^{0}\right), Y\left(z_{1}^{0}, z_{2}^{0}\right)\right)$ gives the field of moduli of the above $(A, \Theta)$. Therefore, by (2.21) and Theorem 2.1 we prove that $K^{*}\left(X\left(z_{1}^{0}, z_{2}^{0}\right), \eta\left(z_{1}^{0}, z_{2}^{0}\right)\right) / K^{*}$ is the class field corresponding to the group $H_{0}$ of (1.7). So, our $(V, \varphi)$ satisfies $(S$-iii).

\section{Cyclic quartic CM fields}

Generically, our class field $K^{*}(X, Y) / K^{*}$ in Theorem 2.1 is not the absolute class field of $K^{*}$, because the group $I_{K^{*}} / H_{0}$ is not equal to the ideal class group $I_{K^{*}} / P_{K^{*}}$. Therefore, it is non-trivial to determine the structure of the class field $K^{*}(X, Y) / K^{*}$. Let us study the detailed structure of $K^{*}(X, Y) / K^{*}$.

In this section, we suppose that $K$ is normal over $\mathbb{Q}$. Then, $\operatorname{Gal}(K / \mathbb{Q})$ is isomorphic to $(\mathbb{Z} / 2 \mathbb{Z})^{2}$ or $\mathbb{Z} / 4 \mathbb{Z}$ (see Example 1.1). However, if $\operatorname{Gal}(K / \mathbb{Q}) \simeq(\mathbb{Z} / 2 \mathbb{Z})^{2}$, then $K$ is not primitive in the sense of Definition 1.2 and $K^{*}$ is an imaginary quadratic field. So, our class field $K^{*}(X, Y) / K^{*}$ does not exceed the scope of Kronecker's Jugendtraum for imaginary quadratic fields.

Hence, in this section, we focus on the case of $\operatorname{Gal}(K / \mathbb{Q}) \simeq \mathbb{Z} / 4 \mathbb{Z}$. Then, according to Example 1.1 (ii), $K^{*}$ coincides with $K$. Based on the results [S4, [HSW], [HW], [HHRWH] and [HHRW] for cyclic extensions, we determine the structure of the Galois group $I_{K} / H_{0}$ of our class field $K(X, Y) / K$.

\subsection{Integral basis of cyclic quartic fields}

Any cyclic quartic field over $\mathbb{Q}$ is given by

$$
K=\mathbb{Q}(\sqrt{A(\Delta+B \sqrt{\Delta})})
$$

where $A, B, C, \Delta \in \mathbb{Z}$ and

$$
\left\{\begin{array}{l}
A \equiv 1 \quad(\bmod 2), \quad \text { squarefree } \\
\Delta=B^{2}+C^{2}, \quad(B>0, C>0), \quad \text { squarefree } \\
\operatorname{GCD}(A, \Delta)=1 .
\end{array}\right.
$$

Let us consider the five cases (i),(ii),(iii),(iv) and (v) (see Table 3).

Proposition 3.1. ([HSW], [HW], [HHRWH] or [HHRW]) Let $K$ be a cyclic quartic field of (3.1).

(1) The conductor of the field $K$ is given by $2^{l} \Delta|A|$, where

$$
l= \begin{cases}3 & (\text { case }(i) \text { and }(i i)), \\ 2 & (\text { case }(\text { iii) }), \\ 0 & (\text { case }(\text { iv }) \text { and }(v)) .\end{cases}
$$




\begin{tabular}{cc}
\hline Case & \\
\hline (i) & $\Delta \equiv 0(\bmod 2)$ \\
(ii) & $\Delta \equiv B \equiv 1(\bmod 2)$ \\
(iii) & $\Delta \equiv 1(\bmod 2), B \equiv 0(\bmod 2), A+B \equiv 3(\bmod 4)$ \\
(iv) & $\Delta \equiv 1(\bmod 2), B \equiv 0(\bmod 2), A+B \equiv 1(\bmod 4), A \equiv C(\bmod 4)$ \\
(v) & $\Delta \equiv 1(\bmod 2), B \equiv 0(\bmod 2), A+B \equiv 1(\bmod 4), A \equiv-C(\bmod 4)$ \\
\hline
\end{tabular}

Table 3: The five cases (i),(ii),(iii),(iv) and (v)

(2) The discriminant of the field $K$ is given by $2^{e} \Delta^{3} A^{2}$, where

$$
e= \begin{cases}8 & (\text { case (i)), } \\ 6 & (\text { case (ii)) } \\ 4 & (\text { case (iii)), } \\ 0 & (\text { case (iv) and (v)) }\end{cases}
$$

(3) Set $\alpha=\sqrt{A(\Delta+B \sqrt{\Delta})}, \beta=\sqrt{A(\Delta-B \sqrt{\Delta})}$. A system of basis of the ring of integers $\mathfrak{O}_{K}$ of $K$ is given by Table 4 .

\begin{tabular}{lc}
\hline Case & Basis \\
\hline (i) & $1, \sqrt{\Delta}, \alpha, \beta$ \\
(ii) & $1, \frac{1+\sqrt{\Delta}}{2}, \alpha, \beta$ \\
(iii) & $1, \frac{1+\sqrt{\Delta}}{2}, \frac{\alpha+\beta}{2}, \frac{\alpha-\beta}{2}$ \\
(iv) & $1, \frac{1+\sqrt{\Delta}}{2}, \frac{1+\sqrt{\Delta}+\alpha+\beta}{4}, \frac{1-\sqrt{\Delta}+\alpha-\beta}{4}$ \\
(v) & $1, \frac{1+\sqrt{\Delta}}{2}, \frac{1+\sqrt{\Delta}+\alpha-\beta}{4}, \frac{1-\sqrt{\Delta}+\alpha+\beta}{4}$ \\
\hline
\end{tabular}

Table 4: The basis of the ring of integers for the cases (i),(ii),(iii),(iv) and (v)

\subsection{Complex tori from cyclic quartic CM fields}

A cyclic quartic field $K$ of (3.1) gives a primitive CM type (see Example 1.1), if $A<0$. We call such fields cyclic quartic CM fields.

In Section 3.1, we obtained a system of basis $\alpha_{1}, \cdots, \alpha_{4}$ of the ring $\mathfrak{O}_{K}$ of integers of $K$. As Section 1.1, we obtain the lattice $\Lambda\left(\mathfrak{O}_{K}\right)$ of $\mathbb{C}^{2}$ from $\alpha_{1}, \cdots, \alpha_{4}$. Then, we have a complex torus $\mathbb{C}^{2} / \Lambda\left(\mathfrak{O}_{K}\right)$. If the Riemann form $E$ of (1.1) on $\Lambda\left(\mathfrak{O}_{K}\right) \times \Lambda\left(\mathfrak{O}_{K}\right)$ is $\mathbb{Z}$-valued, the complex torus $\mathbb{C}^{2} / \Lambda\left(\mathfrak{O}_{K}\right)$ becomes a abelian surface of CM type $\left(\mathfrak{O}_{K},\{i d, \varphi\}\right)$ (see Definition 1.1 and Proposition 1.1).

Recall that the Riemann form of (1.1) depends on $\zeta \in K$ such that $K=K_{0}(\zeta),-\zeta^{2} \in K_{0}$ is totally positive and $\operatorname{Im}\left(\zeta^{\varphi_{j}}\right)>0$. In our study for the field of (3.1), we canonically take

$$
\zeta=\frac{\sqrt{A(\Delta+B \sqrt{\Delta})}}{\kappa}, \quad(\kappa \in \mathbb{Q}) .
$$

Proposition 3.2. For a cyclic field $K$ of (3.1) and a number $\zeta$ of (3.3), the matrix $\left(E\left(\alpha_{j}, \alpha_{k}\right)\right)_{j, k=1, \cdots, 4}$ coming from the alternating Riemann form $E$ of (1.1) is given as Table 5.

Proof. Using Proposition 3.1 (3), we can prove this by a straightforward calculation. 


\begin{tabular}{|c|c|c|c|c|c|c|c|c|c|}
\hline Case & \multicolumn{9}{|c|}{ Matrix $\left(E\left(\alpha_{j}, \alpha_{k}\right)\right)_{j, k=1, \cdots, 4}$} \\
\hline (i) & & & & $-\frac{4 \Delta A}{\kappa}($ & $\left(\begin{array}{c}0 \\
0 \\
-1 \\
0\end{array}\right.$ & $\begin{array}{c}0 \\
0 \\
-B \\
-C\end{array}$ & $\left.\begin{array}{cc}1 & 0 \\
B & C \\
0 & 0 \\
0 & 0\end{array}\right)$ & \multicolumn{2}{|l|}{$\left(=: M_{1}\right)$} \\
\hline (ii) & & & $-\frac{2 \Delta A}{\kappa}$ & $\left(\begin{array}{c}0 \\
0 \\
-2 \\
0\end{array}\right.$ & $\begin{array}{r}0 \\
0 \\
-(1+ \\
-C\end{array}$ & $\begin{array}{l} \\
+B) \\
C\end{array}$ & $\begin{array}{c}2 \\
(1+B) \\
0 \\
0\end{array}$ & $\left.\begin{array}{l}0 \\
C \\
0 \\
0\end{array}\right)\left(=: M_{2}\right)$ & \\
\hline (iii) & & $\frac{\Delta A}{\kappa}$ & $\left(\begin{array}{cc}0 & \\
0 & \\
-2 & -( \\
-2 & -(\end{array}\right.$ & $\begin{array}{c}0 \\
0 \\
1+B+ \\
1+B-\end{array}$ & & $(1+$ & $\begin{array}{l}2 \\
+B+C) \\
0 \\
0\end{array}$ & $\left(\begin{array}{c}2 \\
(1+B-C) \\
0 \\
0\end{array}\right)$ & $\left(=: M_{3}\right)$ \\
\hline (iv) & $\frac{\Delta A}{\kappa}$ & $\left(\begin{array}{c}0 \\
0 \\
-1 \\
-1\end{array}\right.$ & $\begin{array}{l} \\
-(1+ \\
-(1+\end{array}$ & $\begin{array}{l}0 \\
0 \\
B+C) \\
B-C)\end{array}$ & $\begin{array}{l} \\
) / 2 \\
/ 2\end{array}$ & $(1+$ & $\begin{array}{l}1 \\
B+C) / 2 \\
0 \\
-B / 2\end{array}$ & $\begin{array}{c}1 \\
(1+B-C) / 2 \\
B / 2 \\
0\end{array}$ & $2)\left(=: M_{4}\right)$ \\
\hline$(\mathrm{v})$ & $-\frac{\Delta A}{\kappa}$ & $\left(\begin{array}{c}0 \\
0 \\
-1 \\
-1\end{array}\right.$ & $\begin{array}{l} \\
-(1+ \\
-(1+\end{array}$ & $\begin{array}{l}0 \\
0 \\
B-C \\
B+C\end{array}$ & & $(1+$ & $\begin{array}{l}1 \\
B-C) / 2 \\
0 \\
-B / 2\end{array}$ & $\begin{array}{c}1 \\
(1+B+C) / 2 \\
B / 2 \\
0\end{array}$ & $2)\left(=: M_{5}\right)$ \\
\hline
\end{tabular}

Table 5: The matrices for the Riemann form $E$ of (1.1) on $\Lambda\left(\mathfrak{O}_{K}\right) \times \Lambda\left(\mathfrak{O}_{K}\right)$

\subsection{Detailed properties of ideal class groups and Galois groups}

The Galois group for our class field is given by $I_{K} / H_{0}$ (see (1.7)). However, the structure of $H_{0}$ is complicated. To study $H_{0}$, we need some detailed properties of CM fields.

Set

$$
I\left(K / K_{0}\right)=\left\{\mathfrak{a} \in I_{K} \mid \mathfrak{a}\left(\mathfrak{a}^{\rho}\right)^{-1} \in P_{K}\right\}, \quad I_{0}\left(K / K_{0}\right)=P_{K}\left\{\mathfrak{a} \in I_{K} \mid \mathfrak{a}^{\rho}=\mathfrak{a}\right\} .
$$

Let $P_{K_{0}}^{+}$be the subgroup of $P_{K_{0}}$ consisting of all principal ideals generated by totally positive elements. Let $E_{K_{0}}$ be the group of all units in $\mathfrak{O}_{K_{0}}$. Let $E_{K_{0}}^{+}$be the group of all totally positive units in $\mathfrak{O}_{K_{0}}$. Set $E_{K_{0}}^{2}=\left\{u^{2} \mid u \in E_{K_{0}}\right\}$. Then, there exists $\varepsilon \in \mathbb{Z}_{\geq 0}$ such that

$$
2^{\varepsilon}=\left[E_{K_{0}}^{+}: E_{K_{0}}^{2}\right]=\left[P_{K_{0}}: P_{K_{0}}^{+}\right] .
$$

We have the mapping

$$
N_{K / K_{0}}: I_{K} \rightarrow I_{K_{0}}
$$

There exists $\delta \in \mathbb{Z}_{\geq 0}$ such that

$$
2^{\delta}=\left[I_{K_{0}}: P_{K_{0}}^{+} N_{K / K_{0}}\left(I_{K}\right)\right]
$$

Set $R_{K}=\left\{\mathfrak{a} \in I_{K} \mid N_{K / K_{0}}(\mathfrak{a}) \in P_{K_{0}}\right\}$ and $R_{K}^{+}=\left\{\mathfrak{a} \in I_{K} \mid N_{K / K_{0}}(\mathfrak{a}) \in P_{K_{0}}^{+}\right\}$. The mapping $N_{K / K_{0}}$ of (3.6) induces an embedding

$$
I_{K} / R_{K} \hookrightarrow I_{K_{0}} / P_{K_{0}}
$$

Then, we have

$$
\left[R_{K}: R_{K}^{+}\right]=2^{\varepsilon-\delta}
$$


Lemma 3.1. (S4 Appendix) (1) There exist $\eta, \beta, \gamma \in \mathbb{Z}_{\geq 0}$ such that

$$
2^{\eta}=\left[I_{K_{0}} \cap P_{K}: P_{K_{0}}\right], \quad 2^{\beta}=\left[I\left(K / K_{0}\right): I_{0}\left(K / K_{0}\right)\right], \quad 2^{\gamma}=\left[I_{0}\left(K / K_{0}\right): I_{K_{0}} P_{K}\right] .
$$

(2) The relations

$$
\left[I_{K}: I_{K_{0}} P_{K}\right]=2^{\eta} \frac{h_{K}}{h_{K_{0}}}, \quad 2^{\eta-\beta-\gamma} \frac{h_{K}}{h_{K_{0}}}=\left[I_{K}: I\left(K / K_{0}\right)\right], \quad \beta \leq \varepsilon, \quad 2^{\gamma}=2^{\eta+t-1}\left[N_{K / K_{0}}\left(E_{K}\right): E_{K_{0}}^{2}\right] .
$$

hold. Here, $E_{K}$ be the group consists of all units in $\mathfrak{O}_{K}$ and $t$ is the number of prime ideals of $K_{0}$ ramified in $K$.

(3) If $2^{-\eta} h_{K_{0}}$ is an odd number, then

$$
I\left(K / K_{0}\right)=\left\{\mathfrak{a} \in I_{K} \mid \mathfrak{a}^{2} \in I_{K_{0}} P_{K}\right\} .
$$

Moreover, $2^{\beta+\gamma}$ is the number of elements of order 1 or 2 in the ideal class group $I_{K} / P_{K}$.

Let $\left(K,\left\{\varphi_{j}\right\}\right)$ be a CM type and $\left(K^{*},\left\{\psi_{k}\right\}\right)$ be its reflex. In fact, the mapping $\Phi^{*}$ of (1.6) gives a mapping $I_{K^{*}} \rightarrow R_{K}^{+}$. Set

$$
I\left(\Phi^{*}\right)=\left\{\mathfrak{a} \in I_{K^{*}} \mid \mathfrak{a}^{\Phi^{*}} \in P_{K}\right\} .
$$

Lemma 3.2. (ST4 Appendix) There exists an embedding $I\left(\Phi^{*}\right) / H_{0} \hookrightarrow E_{K_{0}}^{+} / N_{K / K_{0}}\left(E_{K}\right)$.

Lemma 3.3. (Sa Appendix) If $K$ is an cyclic extension over $\mathbb{Q}$, then, $K^{*}=K$. Moreover, it holds that $H_{0} \subset I_{0}\left(K / K_{0}\right), I\left(\Phi^{*}\right) \subset I\left(K / K_{0}\right)$ and

$$
I_{K_{0}} P_{K} \subset H_{0} \subset I\left(K / K_{0}\right) \subset I_{K} .
$$

For proofs of the above arguments, see [S4].

Lemma 3.4. Let $K_{0}$ be a totally real number field and $K$ be an imaginary quadratic extension of $K_{0}$. Let us consider the following three conditions:

$$
\begin{cases}(\mathrm{C} 1): & h_{K_{0}}=1 \\ (\mathrm{C} 2): & E_{K_{0}}^{+}=E_{K_{0}}^{2} \\ (\mathrm{C} 3): & K / \mathbb{Q} \text { is cyclic. }\end{cases}
$$

(1) If $K$ satisfies the condition (C1), then $\eta=0$ and $I_{K}=R_{K}$ hold.

(2) If $K$ satisfies the conditions $(\mathrm{C} 1)$ and $(\mathrm{C} 2)$, then $\varepsilon=0, \beta=0, I_{K}=R_{K}^{+}$and $I\left(\Phi^{*}\right)=H_{0}$ hold.

(3) Suppose the ideal class group $I_{K} / P_{K}$ is isomorphic to

$$
(\mathbb{Z} / 2 \mathbb{Z})^{r_{1}} \oplus\left(\mathbb{Z} / 2^{2} \mathbb{Z}\right)^{r_{2}} \oplus \cdots \oplus\left(\mathbb{Z} / 2^{k} \mathbb{Z}\right)^{r_{k}} \oplus G_{1},
$$

where $G_{1}$ does not contain any elements of order $2 m(m \in \mathbb{Z})$. Set $\sum_{j=1}^{k} r_{j}=r$. If $K$ satisfies the conditions $(\mathrm{C} 1),(\mathrm{C} 2)$ and $(\mathrm{C} 3)$, then $\gamma=r,\left[I\left(K / K_{0}\right): P_{K}\right]=2^{r}$ hold.

Proof. (1) Assuming (C1), we have $\eta=0$ and $I_{K}=R_{K}$ from (3.8) and (3.10).

(2) By (C2) and (3.5), we obtain $\varepsilon=0$. From (3.11), $\beta=0$ holds. Moreover, (3.7), (3.9) and the above (1) assure $I_{K}=R_{K}^{+}$. Note that $\left[E_{K_{0}}^{+}: E_{K_{0}}^{2}\right]=\left[E_{K_{0}}^{+}: N_{K / K_{0}}\left(E_{K}\right)\right]\left[N_{K / K_{0}}\left(E_{K}\right): E_{K_{0}}^{2}\right]$. Together with Lemma 3.2, we have $I\left(\Phi^{*}\right)=H_{0}$.

(3) If $I_{K} / P_{K}$ is isomorphic to the group of (3.16), the number of elements of order 1 or 2 of $I_{K} / P_{K}$ is equal to $2^{r}$. By $(\mathrm{C} 1)$ and Lemma 3.1 (3), we have $\beta+\gamma=r$. Together with the above (2), we have $\gamma=r$. So, by (3.10), we obtain $\left[I\left(K / K_{0}\right): P_{K}\right]=2^{r}$. 
Now, we determine the structure of the group $H_{0}$ when $K$ satisfies the conditions $(\mathrm{C} 1),(\mathrm{C} 2)$ and (C3).

Theorem 3.1. Let $K$ be a quartic CM field. Suppose the ideal class group is isomorphic to the group of (3.16). If $K$ satisfies the conditions $(\mathrm{C} 1),(\mathrm{C} 2)$ and $(\mathrm{C} 3)$ in Lemma 3.4, then

$$
I_{K} / H_{0} \simeq(\mathbb{Z} / 2 \mathbb{Z})^{r_{2}} \oplus \cdots \oplus\left(\mathbb{Z} / 2^{k-1} \mathbb{Z}\right)^{r_{k}} \oplus G_{1}
$$

holds.

Proof. Since $K / \mathbb{Q}$ is cyclic, we have

$$
\operatorname{Gal}(K / \mathbb{Q})=\left\{i d, \sigma, \sigma^{2}=\rho, \sigma^{3}\right\},
$$

where $(K,\{i d, \sigma\})$ gives a CM type of $K$ (see Example 1.1). Recall that $(K,\{i d, \sigma\})$ is primitive. The corresponding reflex is given by $\left(K,\left\{i d, \sigma^{-1}\right\}\right)$. The mapping $\Phi^{*}$ of (1.6) has the form

$$
\mathfrak{a} \mapsto \mathfrak{a}^{\Phi^{*}}=\mathfrak{a a d}^{\sigma^{-1}} .
$$

Consider the canonical projection $p: I_{K} \rightarrow I_{K} / P_{K}$ given by $\mathfrak{a} \mapsto[\mathfrak{a}]$. We set $p\left(I\left(K / K_{0}\right)\right)=\overline{I\left(K / K_{0}\right)}$ and $p\left(H_{0}\right)=\overline{H_{0}}$. They are subgroups of the ideal class group $I_{K} / P_{K}$. We shall prove $\overline{H_{0}}=\overline{I\left(K / K_{0}\right)}$.

From Lemma 3.1 (3) (especially (3.12)) and Lemma $3.4(1)$, any $[\mathfrak{b}] \in \overline{I\left(K / K_{0}\right)}$ is a 2-torsion element. Especially, we have

$$
[\mathfrak{b}]^{-1}=[\mathfrak{b}] .
$$

Due to Lemma $3.4(3)$, for some $\left[\mathfrak{b}_{1}\right], \cdots,\left[\mathfrak{b}_{r}\right] \in \overline{I\left(K / K_{0}\right)}$,

$$
\overline{I\left(K / K_{0}\right)}=\left\{\left[\mathfrak{b}_{1}\right]^{e_{1}} \cdots\left[\mathfrak{b}_{r}\right]^{e_{r}} \mid e_{1}, \cdots, e_{r} \in\{0,1\}\right\} \simeq(\mathbb{Z} / 2 \mathbb{Z})^{r},
$$

where $r=\sum_{j=1}^{k} r_{j}$. Here, each factor $(\mathbb{Z} / 2 \mathbb{Z})$ of (3.21) is a subgroup of some $\left(\mathbb{Z} / 2^{j} \mathbb{Z}\right)$ in (3.16). Moreover, according to the definition of $\overline{I\left(K / K_{0}\right)}$ of (3.4), we have

$$
[\mathfrak{b}]^{\rho}=[\mathfrak{b}] \quad\left([\mathfrak{b}] \in \overline{I\left(K / K_{0}\right)}\right) .
$$

On the other hand, by (3.13), Lemma 3.4 (2) and (3.19), we have $\operatorname{Ker}(\Phi)=\bar{H}_{0}$ and

$$
[\mathfrak{a}] \in \overline{H_{0}} \Longleftrightarrow[\mathfrak{a}]^{\sigma}=[\mathfrak{a}]^{-1} \text {. }
$$

Under the condition (C1), the sequence (3.14) becomes to be $P_{K} \subset H_{0} \subset I\left(K / K_{0}\right) \subset I_{K}$. Let us assume

$$
\overline{H_{0}} \varsubsetneqq \overline{I\left(K / K_{0}\right)} .
$$

Then, without loss of generality, we can take $\left[\mathfrak{b}_{1}\right], \cdots,\left[\mathfrak{b}_{r}\right]$ in $(3.21)$ and $s \in \mathbb{Z}(0<s \leq r)$ such that

$$
\overline{H_{0}}=\left\{\left[\mathfrak{b}_{s+1}\right]^{e_{s+1}} \cdots\left[\mathfrak{b}_{r}\right]^{e_{r}} \mid e_{s+1}, \cdots, e_{r} \in\{0,1\}\right\} .
$$

So, according to (3.21), $J_{0}=\overline{I\left(K / K_{0}\right)} / \overline{H_{0}}$ must be in the form

$$
J_{0}=\left\{\left[\mathfrak{b}_{1}\right]^{e_{1}} \cdots\left[\mathfrak{b}_{s}\right]^{e_{s}} \mid e_{1}, \cdots, e_{s} \in\{0,1\}\right\} .
$$

For any $[\mathfrak{b}] \in \overline{I\left(K / K_{0}\right)}$, we have $[\mathfrak{b}]^{\sigma} \in \overline{I\left(K / K_{0}\right)}$ since $\left([\mathfrak{b}]^{\sigma}\right)^{\rho}=\left([\mathfrak{b}]^{\rho}\right)^{\sigma}=[\mathfrak{b}]^{\sigma}$. By virtue of $(3.23)$, we have

$$
\left[\mathfrak{b}_{j}\right]^{\sigma} \in J_{0}, \quad\left[\mathfrak{b}_{j}\right]^{\sigma} \neq\left[\mathfrak{b}_{j}\right] \quad(j \in\{1, \cdots, s\}) .
$$


For $j \in\{1, \cdots, s\}$, set

$$
\left[\mathfrak{c}_{j}\right]=\left[\mathfrak{b}_{j}\right]\left[\mathfrak{b}_{j}\right]^{\sigma}\left(\neq i d \overline{I\left(K / K_{0}\right)}\right) .
$$

According to (3.25) and (3.26), $\left[\mathfrak{c}_{j}\right] \in J_{0}$. From (3.18) and (3.22), we have

$$
\left[\mathfrak{c}_{j}\right]^{\sigma}=\left(\left[\mathfrak{b}_{j}\right]\left[\mathfrak{b}_{j}\right]^{\sigma}\right)^{\sigma}=\left[\mathfrak{b}_{j}\right]^{\sigma}\left[\mathfrak{b}_{j}\right]^{\rho}=\left[\mathfrak{b}_{j}\right]^{\sigma}\left[\mathfrak{b}_{j}\right]=\left[\mathfrak{c}_{j}\right] .
$$

Therefore, $\left[\mathfrak{c}_{j}\right] \in \overline{H_{0}}$. This is a contradiction.

Hence, the assumption (3.24) is not true. Therefore, we have $\overline{I\left(K / K_{0}\right)}=\overline{H_{0}}$. According to (3.16) and (3.21), we have (3.17).

Corollary 3.1. Let $K$ be a quartic $C M$ field as in Theorem 3.1. Set $r=\sum_{j=1}^{k} r_{j}$. The unramified class field $K(X, Y) / K$ corresponding to the group $H_{0}$ of 1.7$)$ is an extension over $K$ of degree $\frac{h_{K}}{2^{r}}$.

Proof. It is clear because we have Theorem 1.1 and Theorem 3.1

\subsection{Cyclic quartic CM fields over $\mathbb{Q}(\sqrt{5})$}

Letting $K_{0}$ be a real quadratic field, the group $E_{K_{0}}$ of units in $\mathfrak{O}_{K_{0}}$ is given by the direct product of $\{i d,-i d\}$ and $\left\{\varepsilon_{0}^{n} \mid n \in \mathbb{Z}\right\}$. Here, $\varepsilon_{0}$ is called a fundamental unit of $K_{0}$. If $N_{K / K_{0}}\left(\varepsilon_{0}\right)=-1$, then the group $E_{K_{0}}^{+}$of totally positive units in $\mathfrak{O}_{K_{0}}$ is given by $\left\{\varepsilon_{0}^{2 n} \mid n \in \mathbb{Z}\right\}$ and we have $E_{K_{0}}^{+}=E_{K_{0}}^{2}$.

In this section, we study the case for $K_{0}=\mathbb{Q}(\sqrt{5})$ in detail. The class number of $\mathbb{Q}(\sqrt{5})$ is equal to 1. A fundamental unit of $\mathbb{Q}(\sqrt{5})$ is given by $\frac{1-\sqrt{5}}{2}$, that satisfies $N_{\mathbb{Q}(\sqrt{5}) / \mathbb{Q}}\left(\frac{1-\sqrt{5}}{2}\right)=-1$. Therefore, if $K$ is an imaginary quadratic extension over $\mathbb{Q}(\sqrt{5})$, then $K$ satisfies the conditions $(\mathrm{C} 1)$ and $(\mathrm{C} 2)$ in Lemma 3.4 Moreover, if $K / \mathbb{Q}$ is cyclic and $K / \mathbb{Q}(\sqrt{5})$ is totally imaginary quadratic, it follows that

$$
(B, C)=(1,2) \text { or }(2,1)
$$

(see (3.2)).

Theorem 3.2. Let $K$ of (3.1) be a totally imaginary extension of $\mathbb{Q}(\sqrt{5})$ such that $K / \mathbb{Q}$ is cyclic. Set $\alpha=\sqrt{A(5+B \sqrt{5})}$. The Riemann form $E$ of (1.1) given by $\zeta$ in Table 6 gives a principal polarization on the abelian variety $A\left(\mathfrak{O}_{K}\right)$.

\begin{tabular}{cc}
\hline Case & $\zeta$ of (3.3) \\
\hline (ii) & $\frac{\alpha}{-4 \Delta A}=\frac{\alpha}{-20 A}$ \\
(iii) $\frac{\alpha}{-2 \Delta A}=\frac{\alpha}{-10 A}$ \\
(iv) $\frac{\alpha}{-\Delta A}=\frac{\alpha}{-5 A}$ \\
(v) $\frac{\alpha}{-\Delta A}=\frac{\alpha}{-5 A}$ \\
\hline
\end{tabular}

Table 6: The number $\zeta$ which gives a principal polarization for $\Delta=5$

Proof. If the alternating Riemann form $E$ of (1.1) is $\mathbb{Z}$-valued, the determinant of the matrix $M_{j}$ in Table 5 is a perfect square number. The positive root of this determinant is called the Pfaffian of $E$. If the Pfaffian of $E$ is equal to 1, then the Riemann form $E$ induces a principal polarization on the complex torus $\mathbb{C}^{2} / \Lambda\left(\mathfrak{O}_{K}\right)=A\left(\mathfrak{O}_{K}\right)$. Hence, it is sufficient to see that the number $\zeta$ in Table 6 gives the $\mathbb{Z}$-valued matrix $M_{j}$ in Table 5 and the determinant of $M_{j}$ is equal to 1. 
(ii) In this case, we consider the matrix $M_{2}$ in Table 5 . Putting $(B, C)=(1,2)$ and $\kappa=-4 \Delta A$, we have

$$
M_{2}=\left(\begin{array}{cccc}
0 & 0 & 1 & 0 \\
0 & 0 & 1 & 1 \\
-1 & -1 & 0 & 0 \\
0 & -1 & 0 & 0
\end{array}\right)
$$

(iii) In this case, we consider the matrix $M_{3}$ in Table 5 . Putting $(B, C)=(2,1)$ and $\kappa=-2 \Delta A$, we have

$$
M_{3}=\left(\begin{array}{cccc}
0 & 0 & 1 & 1 \\
0 & 0 & 2 & 1 \\
-1 & -2 & 0 & 0 \\
-1 & -1 & 0 & 0
\end{array}\right)
$$

(iv) In this case, we consider the matrix $M_{4}$ in Table 5 . If we consider the case (iv), putting $(B, C)=$ $(1,2)$ and $\kappa=-\Delta A$, we have

$$
M_{4}=\left(\begin{array}{cccc}
0 & 0 & 1 & 1 \\
0 & 0 & 2 & 1 \\
-1 & -2 & 0 & 1 \\
-1 & -1 & -1 & 0
\end{array}\right)
$$

(v) In this case, we consider the matrix $M_{5}$ in Table 5 . If we consider the case (v), putting $(B, C)=$ $(1,2)$ and $\kappa=-\Delta A$, , we have

$$
M_{5}=\left(\begin{array}{cccc}
0 & 0 & 1 & 1 \\
0 & 0 & 1 & 2 \\
-1 & -1 & 0 & 1 \\
-1 & -2 & -1 & 0
\end{array}\right)
$$

Theorem 3.2 implies that we only need principally polarized abelian surfaces to construct unramified class fields $k_{0}$ in Section 1.4 for $\Delta=5$. Hence, Theorem 2.1 is available for every cyclic quartic CM field $K$ for $\Delta=5$.

Remark 3.1. The construction of class fields in Section 1.4 for $\Delta=5$ is simpler than the cases of $\Delta>5$. In general, in those cases we need non-principally polarized abelian surfaces.

\subsection{Examples}

In this section, we apply our results of the icosahedral invariants $X$ and $Y$ to concrete cyclic CM fields $K$. We construct unramified class fields $k_{0}$ in Section 1.4 over cyclic quartic CM fields $K$ with the maximal real subfield $\mathbb{Q}(\sqrt{5})$. According to Theorem 2.1 and Theorem 3.2. such class fields are given by the special values of $X$ and $Y$ of (2.8).

Hardy, Hudson, Richmann, Williams and Hiltz HHRWH listed imaginary cyclic quartic fields $K$ with small conductor. Using their result, we will obtain examples of our construction of class fields. In Section 3.5.1, we shall give an example such that $\left[k_{0}: K\right]=1$. In Section 3.5.2, we shall give an example such that $\left[k_{0}: K\right]=5>1$.

\subsubsection{Case $K=\mathbb{Q}(\sqrt{-(5+\sqrt{5})})$}

Let us take $K=\mathbb{Q}(\sqrt{-(5+\sqrt{5})})$. From [HHRWH] , the conductor of $K$ is 5 . We consider the case (ii) in Table 3.

According to Theorem 3.2 taking $\zeta=\frac{\sqrt{-(5+\sqrt{5})}}{20}$, we have the Riemann form $E$ of (1.1). Next, we take a system $\left\{\alpha_{1}, \cdots, \alpha_{4}\right\}$ of basis as in Table 4 . Then, we have the lattice $\Lambda=\left\langle u\left(\alpha_{1}\right), \cdots, u\left(\alpha_{4}\right)\right\rangle$ of 
$\mathbb{C}^{2}$, where

$$
\left\{\begin{array}{l}
u\left(\alpha_{1}\right)=\left(\begin{array}{l}
1 \\
1
\end{array}\right), \quad u\left(\alpha_{2}\right)=\left(\begin{array}{c}
\frac{1+\sqrt{5}}{2} \\
\frac{1-\sqrt{5}}{2}
\end{array}\right) \\
u\left(\alpha_{3}\right)=\left(\begin{array}{l}
\sqrt{-(5+\sqrt{5})} \\
\sqrt{-(5-\sqrt{5})}
\end{array}\right), \quad u\left(\alpha_{4}\right)=\left(\begin{array}{c}
\sqrt{-(5-\sqrt{5})} \\
-\sqrt{-(5+\sqrt{5})}
\end{array}\right) .
\end{array}\right.
$$

Putting $\lambda_{1}=u\left(\alpha_{1}\right), \lambda_{2}=u\left(\alpha_{2}\right), \lambda_{3}=u\left(\alpha_{3}\right)-u\left(\alpha_{4}\right)$ and $\lambda_{4}=u\left(\alpha_{4}\right)$, we have

$$
E\left(\lambda_{j}, \lambda_{k}\right)=\left(\begin{array}{cccc}
0 & 0 & 1 & 0 \\
0 & 0 & 0 & 1 \\
-1 & 0 & 0 & 0 \\
0 & -1 & 0 & 0
\end{array}\right)
$$

We set $\left(M_{1} M_{2}\right)=\left(\lambda_{1} \lambda_{2} \lambda_{3} \lambda_{4}\right)$, where $M_{1}, M_{2} \in M(2, \mathbb{C})$. So, we have $\Omega=-M_{2}^{-1} M_{1} \in \mathfrak{S}_{2}$.

$$
\operatorname{Set}\left(\begin{array}{ll}
A_{0} & B_{0} \\
C_{0} & D_{0}
\end{array}\right)=\left(\begin{array}{llll}
0 & 1 & 0 & 0 \\
1 & 0 & 0 & 0 \\
0 & 0 & 0 & 1 \\
0 & 0 & 1 & 0
\end{array}\right) \in S p(2, \mathbb{Z}) .
$$

Then, we have $\tilde{\Omega}=\left(A_{0} \Omega+B_{0}\right)\left(C_{0} \Omega+D_{0}\right)^{-1}=\left(\begin{array}{ll}\tau_{1} & \tau_{2} \\ \tau_{2} & \tau_{3}\end{array}\right)$, where $\tau_{1}=\frac{1}{5} \sqrt{-1} \sqrt{\frac{25}{2}+\sqrt{5}}, \tau_{2}=$ $\frac{1}{5} \sqrt{-1} \sqrt{5-\sqrt{5}}$ and $\tau_{3}=\frac{1}{5} \sqrt{-1} \sqrt{\frac{5}{2}+\sqrt{5}}$. This satisfies $\tau_{1}-\tau_{2}-\tau_{3}=0$. So, from $\tau_{2}$ and $\tau_{3}$, we have $\left(z_{1}^{0}, z_{2}^{0}\right) \in \mathbb{H} \times \mathbb{H}$ as in (2.18). According to Theorem 2.1] using the pair of modular functions $(X, Y)$ of (2.8), the unramified class field $k_{0}$ over $K=\mathbb{Q}(\sqrt{-(5+\sqrt{5})})$ corresponding to the group $H_{0}$ of (1.7) is given by $k_{0}=K\left(X\left(z_{1}^{0}, z_{2}^{0}\right), Y\left(z_{1}^{0}, z_{2}^{0}\right)\right)$.

Due to [HHRWH], the class number $h_{K}$ of $K=\mathbb{Q}(\sqrt{-(5+\sqrt{5})})$ is equal to 2. Therefore, $I_{K} / P_{K}$ is isomorphic to $(\mathbb{Z} / 2 \mathbb{Z})$. Applying Theorem 3.1 to this case, we have $\left[k_{0}: K\right]=1$. This case does not give a non-trivial class-field over the CM field $K$.

Remark 3.2. In fact, Murabayashi and Umegaki [MU] proved that the field of moduli of principally polarized abelian surface corresponding to $K=\mathbb{Q}(\sqrt{-(5+\sqrt{5})})$ coincides with $\mathbb{Q}$. So, our result does not contradict their result.

Remark 3.3. In this case, since $h_{K}=2$, there exists the absolute class field $C(K)$ over $K$ such that $[C(K): K]=2$. In [NS2] Example 5.2, we have an explicit construction of $C(K) / K$ using another explicit modular function derived from the moduli of a PEL-structure for 8-dimensional abelian varieties.

3.5.2 Case $K=\mathbb{Q}(\sqrt{-37(5+2 \sqrt{5})})$

Let us take $K=\mathbb{Q}(\sqrt{-37(5+2 \sqrt{5})})$. From [HHRWH, the conductor of $K$ is 185 . We consider the case (v) in Table 3.

According to Theorem 3.2 taking $\zeta=\frac{\sqrt{-37(5+2 \sqrt{5})}}{185}$, we have the Riemann form $E$ of (1.1). Next, we take a system $\left\{\alpha_{1}, \cdots, \alpha_{4}\right\}$ of basis as in Table 4 . Then, we have the lattice $\Lambda=\left\langle u\left(\alpha_{1}\right), \cdots, u\left(\alpha_{4}\right)\right\rangle$ of 
$\mathbb{C}^{2}$, where

$$
\left\{\begin{array}{l}
u\left(\alpha_{1}\right)=\left(\begin{array}{l}
1 \\
1
\end{array}\right), \quad u\left(\alpha_{2}\right)=\left(\begin{array}{l}
\frac{1+\sqrt{5}}{2} \\
\frac{1-\sqrt{5}}{2}
\end{array}\right) \\
u\left(\alpha_{3}\right)=\frac{1}{4}\left(\begin{array}{l}
1+\sqrt{5}+\sqrt{-37(5+2 \sqrt{5})}-\sqrt{-37(5-2 \sqrt{5})} \\
1-\sqrt{5}+\sqrt{-37(5-2 \sqrt{5})}+\sqrt{-37(5+2 \sqrt{5})}
\end{array}\right) \\
u\left(\alpha_{4}\right)=\frac{1}{4}\left(\begin{array}{l}
1-\sqrt{5}+\sqrt{-37(5+2 \sqrt{5})}+\sqrt{-37(5-2 \sqrt{5})} \\
1+\sqrt{5}+\sqrt{-37(5-2 \sqrt{5})}-\sqrt{-37(5+2 \sqrt{5})}
\end{array}\right)
\end{array} .\right.
$$

Putting $\lambda_{1}=u\left(\alpha_{1}\right), \lambda_{2}=u\left(\alpha_{2}\right), \lambda_{3}=2 u\left(\alpha_{3}\right)-u\left(\alpha_{4}\right)$ and $\lambda_{4}=u\left(\alpha_{4}\right)-u\left(\alpha_{3}\right)+u\left(\alpha_{1}\right)$, we have

$$
E\left(\lambda_{j}, \lambda_{k}\right)=\left(\begin{array}{cccc}
0 & 0 & 1 & 0 \\
0 & 0 & 0 & 1 \\
-1 & 0 & 0 & 0 \\
0 & -1 & 0 & 0
\end{array}\right) .
$$

We set $\left(M_{1} M_{2}\right)=\left(\lambda_{1} \lambda_{2} \lambda_{3} \lambda_{4}\right)$, where $M_{1}, M_{2} \in M(2, \mathbb{C})$. So, we have $\Omega=-M_{2}^{-1} M_{1} \in \mathfrak{S}_{2}$.

$$
\text { Set }\left(\begin{array}{ll}
A_{0} & B_{0} \\
C_{0} & D_{0}
\end{array}\right)=\left(\begin{array}{cccc}
0 & -1 & 0 & 1 \\
1 & 0 & 1 & 0 \\
0 & -1 & 0 & 0 \\
-1 & 0 & 0 & 0
\end{array}\right) \in S p(2, \mathbb{Z}) \text {. Then, we have } \tilde{\Omega}=\left(A_{0} \Omega+B_{0}\right)\left(C_{0} \Omega+D_{0}\right)^{-1}=
$$

$\left(\begin{array}{ll}\tau_{1} & \tau_{2} \\ \tau_{2} & \tau_{3}\end{array}\right)$, where $\tau_{1}=\sqrt{-1} \sqrt{\frac{37}{10}(5+\sqrt{5})}, \tau_{2}=\frac{3}{2}-\frac{1}{2} \sqrt{-1} \sqrt{185-\frac{74}{\sqrt{5}}}$ and $\tau_{3}=-\frac{3}{2}+\frac{1}{2} \sqrt{-1} \sqrt{481+\frac{74}{\sqrt{5}}}$. This satisfies $\tau_{1}-\tau_{2}-\tau_{3}=0$. So, from $\tau_{2}$ and $\tau_{3}$, we have $\left(z_{1}^{0}, z_{2}^{0}\right) \in \mathbb{H} \times \mathbb{H}$ as in (2.18). According to Theorem 2.1, using the pair of modular functions $(X, Y)$ of (2.8), the unramified class field $k_{0}$ over $K=$ $\mathbb{Q}(\sqrt{-37(5+2 \sqrt{5})})$ corresponding to the group $H_{0}$ of (1.7) is given by $k_{0}=K\left(X\left(z_{1}^{0}, z_{2}^{0}\right), Y\left(z_{1}^{0}, z_{2}^{0}\right)\right)$.

Due to [HHRWH], the class number $h_{K}$ of $K=\mathbb{Q}(\sqrt{-37(5+2 \sqrt{5})})$ is equal to 10. Therefore, $I_{K} / P_{K}$ is isomorphic to $(\mathbb{Z} / 2 \mathbb{Z}) \oplus(\mathbb{Z} / 5 \mathbb{Z})$. Applying Theorem 3.1 to this case, we have $\left[k_{0}: K\right]=5$. Namely, this case gives an example of an unramified class field $k_{0}$ corresponding to $H_{0}$ of (1.7) which gives a non-trivial extension $k_{0} / K$.

\section{Acknowledgment}

The author would like to thank Professor Hironori Shiga for helpful advises and valuable suggestions, and also to Professor Kimio Ueno for kind encouragements. This work is supported by The JSPS Program for Advancing Strategic International Networks to Accelerate the Circulation of Talented Researchers "Mathematical Science of Symmetry, Topology and Moduli, Evolution of International Research Network based on OCAMI", The Sumitomo Foundation Grant for Basic Science Research Project (No.150108) and Waseda University Grant for Special Research Project (2014B-169 and 2015B-191).

\section{References}

[CD] A. Clinger and C. Doran, Lattice polarized K3 surfaces and Siegel modular forms, Adv. Math. 231, 2012, 172-212.

[EK] N. Elkies and A. Kumar, K3 Surfaces and equations for Hilbert modular surfaces, Algebra Number Theory 8, 2014, 2297-2411.

[FG] Y. Fuertes and G. González-Diez, Fields of moduli and definition of hyperelliptic covers, Arch. Math. 86 (5), 2006, 398-408.

[Ge] G. van der Geer, Hilbert Modular Surfaces, Ergebnisse der Mathematik und ihrer Grenzgebiete, SpringerVerlag, 1988. 
[Gj] K. Gunji, Defining equations of the universal abelian surfaces with level three structure, Manuscripta Math. 119, 2006, 61-96.

[Gl] K. Gundlach, Die Bestimmung der Funktionen zur Hilbertschen Modulgruppe des Zahlköpers $Q(\sqrt{5})$, Math. Ann. 152, 1963, 226-256.

[H] F. Hirzebruch, The ring of Hilbert modular forms for real quadratic fields of small discriminant, Lecture Notes in Math. 627, Springer-Verlag, 1977, 287-323.

[HHRWH] K. Hardy, R. H. Hudson, D. Richmann, K. S. Williams and N. M. Holtz, Calculation of Class Numbers of Imaginary Cyclic Quartic Fields, Math. Computation 49, 1987, 615-620.

[HHRW] K. Hardy, R. H. Hudson, D. Richmann and K. S. Williams, Determination of all Imaginary Cyclic Quartic Fields with Class Number 2, Trans. Amer. Math., 1989, 1-55.

[HNU] K. Hashimoto, A. Nagano and K. Ueda, Modular surfaces associated with toric K3 surfaces, arXiv:1403.5818, 2014.

[HSW] J. G. Huard, B. K. Spearman and K. S. Williams, Integral basis for quartic fields with quadratic subfields, J. Number Theory 51, 1995, 87-102.

[HW] R. H. Hudson and K. S. Williams, The integers of a cyclic quartic field, Rocky Mountain J. Math. 20, 1990, 145-150.

[I] J. Igusa, Arithmetic variety of moduli for genus two, Ann. Math, 72 (3), 1960, 612-649.

[K] A. Kumar, K3 surfaces associated to curves of genus two, Int. Math. Re. Not. 16 (2008), ArticleID: rnm165.

[LY] K. Lauter and T. H. Yang, Computing genus 2 curves from invariants on the Hilbert moduli space, J. Number Theory 131, 2011, 936-958.

[K] F. Klein, Vorlesungen über das Ikosaeder und die Auflösung der Gleichungen vom fünften Grade, Tauber, 1884.

[Mul] R. Müller Hilbertsche Modulformen und modulfunktionen zu $\mathbb{Q}(\sqrt{5})$, Arch. Math. 45, 1985, $239-251$.

[Mum] D. Mumford, On the equations defining abelian varieties I, Invent. Math. 1, 1967, 287-354.

[MU] N. Murabayashi and A. Umegaki, Determination of all $\mathbb{Q}$-rational CM-points in the moduli space of principally polarized abelian surfaces, J. Alg. 235, 2001, 267-274.

[NS1] A. Nagano and H. Shiga, Modular map for the family of abelian surfaces via elliptic K3 surfaces, Math. Nachr. 288, 2014, 89-114.

[NS2] A. Nagano and H. Shiga, To the Hilbert class field from the hypergeometric modular function, J. Number Theory 165, 2016, 408-430.

[N1] A. Nagano, A theta expression of the Hilbert modular functions for $\sqrt{5}$ via period of K3 surfaces, Kyoto J. Math. 53 (4), 2013, 815-843.

[N2] A. Nagano, Double integrals on a weighted projective plane and the Hilbert modular functions for $\mathbb{Q}(\sqrt{5})$, Acta Arith. 167, 2015, 327-345.

[N3] A. Nagano, Icosahedral invariants and Shimura curves, J. Theor. Nom. Bordeaux, 2017, to appear.

[S1] G. Shimura, On the theory of automorphic functions, Ann. of Math. 70, 1959, 101-144.

[S2] G. Shimura, On purely transcendental fields of automorphic functions of several variables, Osaka J. Math. 1, 1963, 1-14.

[S3] G. Shimura, Construction of class fields and zeta functions of algebraic curves, Ann. of Math. 85, 1967, 58-159.

[S4] G. Shimura, On abelian varieties with complex multiplication, Proc. London. Math. Soc. 34 (3), $1977,65-86$.

[S5] G. Shimura, Abelian Varieties with Complex Multiplication and Modular Functions, Princeton Univ. Press, 1997.

[W] A. Weil, On the theory of complex multiplication, Proc. International Symposium Algebraic Number Theory, 1955, 9-22.

\author{
Atsuhira Nagano \\ Department of Mathematics \\ King's College London \\ Strand, London, WC2R 2LS \\ The United Kingdom \\ (E-mail: atsuhira.nagano@gmail.com)
}

